\title{
SUFFICIENT SECOND-ORDER CONDITIONS FOR BANG-BANG CONTROL PROBLEMS*
}

\author{
EDUARDO $\mathrm{CASAS}^{\dagger}$, DANIEL WACHSMUTH ${ }^{\ddagger}$, AND GERD WACHSMUTH ${ }^{\S}$
}

\begin{abstract}
We provide sufficient optimality conditions for optimal control problems with bangbang controls. Building on a structural assumption on the adjoint state, we additionally need a weak second-order condition. This second-order condition is formulated with functions from an extended critical cone, and it is equivalent to a formulation posed on measures supported on the set where the adjoint state vanishes. If our sufficient optimality condition is satisfied, we obtain a local quadratic growth condition in $L^{1}(\Omega)$.
\end{abstract}

Key words. bang-bang control, second-order conditions, sufficient optimality conditions, critical cone

AMS subject classifications. 49K20, 49K30, 35J61

DOI. $10.1137 / 16 \mathrm{M} 1099674$

1. Introduction. The aim of the present paper is to provide second-order sufficient conditions for bang-bang optimal control problems with nonlinear partial differential equations (PDEs). In particular, we consider the case that the control variable does not appear explicitly in the objective. The control problem we are interested in is given as follows:

$$
\begin{cases}\text { Minimize } & J(u):=\int_{\Omega} L\left(x, y_{u}(x)\right) \mathrm{d} x \\ \text { such that } & \alpha(x) \leq u(x) \leq \beta(x) \text { for a.a. } x \in \Omega,\end{cases}
$$

where $y_{u}$ is the weak solution of the elliptic boundary value problem

$$
\left\{\begin{array}{rlr}
A y+a(\cdot, y)=u & & \text { in } \Omega, \\
y=0 & & \text { on } \Gamma .
\end{array}\right.
$$

The assumptions on the various ingredients are specified below in section 2. Under these assumptions, solutions of our problem (P) exist. If $\bar{u}$ is a locally optimal control with associated state $\bar{y}:=y_{\bar{u}}$, then there exists an adjoint state $\bar{\varphi}$ such that

$$
\bar{u}(x)= \begin{cases}\alpha(x) & \text { if } \bar{\varphi}(x)>0, \\ \beta(x) & \text { if } \bar{\varphi}(x)<0 .\end{cases}
$$

In the case that $\bar{\varphi} \neq 0$ holds a.e. in $\Omega$, the control $\bar{u}$ attains the control bounds a.e. Hence, $\bar{u}$ is a bang-bang control. Naturally, we arrive at the question of whether we can

\footnotetext{
* Received by the editors October 19, 2016; accepted for publication (in revised form) July 17, 2017; published electronically September 28, 2017.

http://www.siam.org/journals/sicon/55-5/M109967.html

Funding: The first author was partially supported by the Spanish Ministerio de Economía y Competitividad under project MTM2014-57531-P. The second author was partially supported by the DFG under grant Wa 3626/1-1.

$\dagger$ Departmento de Matemática Aplicada y Ciencias de la Computación, E.T.S.I. Industriales y de Telecomunicación, Universidad de Cantabria, 39005 Santander, Spain (eduardo.casas@unican.es).

${ }^{\ddagger}$ Institut für Mathematik, Universität Würzburg, 97074 Würzburg, Germany (daniel.wachsmuth@ mathematik.uni-wuerzburg.de).

$\S$ Faculty of Mathematics, Technische Universität Chemnitz, 09107 Chemnitz, Germany (gerd. wachsmuth@mathematik.tu-chemnitz.de).
} 
find sufficient optimality conditions, i.e., conditions which imply that a certain control is locally optimal. As the objective functional $J$ depends only implicitly through $y_{u}$ on the control $u$, standard sufficient second-order conditions, which require the coercivity of $J^{\prime \prime}$ in $L^{2}(\Omega)$, cannot be satisfied. Hence, second-order conditions tailored towards the special problem structure need to be devised.

The only contribution which deals with this situation in the PDE constrained case is [6]. Therein, the author provided second-order conditions involving coercivity w.r.t. the linearized state. It is shown that these conditions imply local quadratic growth of $J$ w.r.t. the linearized state in a neighborhood of $\bar{u}$.

In the literature on control problems governed by ordinary differential equations (ODEs) there are many contributions dealing with second-order conditions in the bang-bang case; see, e.g., $[11,13,14,17,18,19,20]$. In these contributions one typically assumes that the (differentiable) switching function $\sigma:[0, T] \rightarrow \mathbb{R}$ possesses only finitely many zeros and that $|\dot{\sigma}(t)|>0$ is satisfied for all zeros $t$ of $\sigma$. This condition cannot be transferred to the PDE constrained case, in which the role of the switching function is played by the distributed adjoint state $\bar{\varphi}: \Omega \rightarrow \mathbb{R}$. It is not hard to check that this condition on the switching implies the existence of $K>0$ such that

$$
|\{t \in[0, T]:|\sigma(t)| \leq \varepsilon\}| \leq K \varepsilon
$$

holds for all $\varepsilon>0$. Here, $|\cdot|$ denotes the Lebesgue measure. This latter condition easily generalizes to the PDE constrained case; namely, one assumes that

$$
|\{x \in \Omega:|\bar{\varphi}(x)| \leq \varepsilon\}| \leq K \varepsilon
$$

is satisfied, where $\bar{\varphi}: \Omega \rightarrow \mathbb{R}$ is the adjoint state associated with a fixed control $\bar{u}$. In the PDE constrained case, the condition (1.2) was already successfully used in [8] to study the discretization error in the bang-bang case and in [26] to obtain regularization error estimates for bang-bang controls.

The regularity condition (1.2) on the adjoint state is our starting point to derive second-order sufficient conditions for bang-bang controls. In Proposition 2.7, we show the existence of $\kappa>0$ such that

$$
J^{\prime}(\bar{u})(u-\bar{u}) \geq \kappa\|u-\bar{u}\|_{L^{1}(\Omega)}^{2} \quad \forall u \in \mathcal{U}_{\mathrm{ad}} .
$$

Due to this quadratic growth estimate, it turns out that we can prove local optimality of $\bar{u}$ if $J^{\prime \prime}$ is bounded from below on certain critical cones. We show that the condition

$$
J^{\prime \prime}(\bar{u}) v^{2} \geq-\kappa^{\prime}\|v\|_{L^{1}(\Omega)}^{2} \forall v \in C_{\bar{u}}^{\tau} \subset L^{2}(\Omega)
$$

with $\kappa^{\prime}<\kappa$ and $\tau>0$ yields local optimality of $\bar{u}$ in $L^{1}(\Omega)$ (see Theorem 2.8), and this is a main result of this work. The support of functions from $C_{\bar{u}}^{\tau}$ is contained in $\{x \in \Omega:|\bar{\varphi}(x)| \leq \tau\}$. Remarkably, it is impossible to allow $\tau=0$, as $C_{\bar{u}}^{0}=\{0\}$ is just the trivial cone for bang-bang $\bar{u}$. To circumvent this difficulty we consider cones of measures with support on the set $\{x \in \Omega: \bar{\varphi}(x)=0\}$; see subsection 2.5. We prove the following. Suppose that

$$
J^{\prime \prime}(\bar{u}) \mu^{2} \geq-\kappa^{\prime}\|\mu\|_{\mathcal{M}(\Omega)}^{2}
$$

holds for all measures $\mu$ supported on $\{x \in \Omega: \bar{\varphi}(x)=0\}$ and satisfying certain sign conditions. Then, $\bar{u}$ is locally optimal and satisfies a quadratic growth condition w.r.t. $L^{1}(\Omega)$; see Corollary 2.15 . 
We expected that condition (1.4) involving measures with support on $\{x \in \Omega$ : $\bar{\varphi}(x)=0\}$ is weaker than condition (1.3), which involves $L^{1}$-functions supported on the larger set $\{x \in \Omega:|\bar{\varphi}(x)| \leq \tau\}$. However, it turns out that both conditions are equivalent (see Theorem 2.14), which can be regarded as one of the main results of our article.

We emphasize that the second-order conditions (1.3) and (1.4) are rather weak, as they allow for some negative curvature of the reduced objective.

Naturally, these considerations can be transferred to different situations. We discuss them in an abstract setting in section 3 .

\section{An elliptic bang-bang control problem.}

2.1. Preliminary results. Let us first state the standing assumptions on the data of the control problem $(\mathrm{P})$ and in particular of the state equation (2.1). We assume $\Omega \subset \mathbb{R}^{n}, n \in\{1,2,3\}, \alpha, \beta \in L^{\infty}(\Omega), \alpha \leq \beta$, and $\alpha \not \equiv \beta$. Moreover, $L, a: \Omega \times \mathbb{R} \longrightarrow \mathbb{R}$ are Carathéodory functions of class $C^{2}$ w.r.t. the second variable such that the following assumptions are satisfied:

(A1) $a(\cdot, 0) \in L^{\bar{p}}(\Omega)$, with $\bar{p}>n / 2$,

$$
\frac{\partial a}{\partial y}(x, y) \geq 0 \quad \text { for a.a. } x \in \Omega \text { and } \forall y \in \mathbb{R},
$$

and for all $M>0$ there exists a constant $C_{a, M}>0$ such that

$$
\left|\frac{\partial a}{\partial y}(x, y)\right|+\left|\frac{\partial^{2} a}{\partial y^{2}}(x, y)\right| \leq C_{a, M} \text { for a.e. } x \in \Omega \text { and } \forall|y| \leq M \text {. }
$$

For every $M>0$ and $\varepsilon>0$ there exists $\delta>0$, depending on $M$ and $\varepsilon$, such that

$$
\left|\frac{\partial^{2} a}{\partial y^{2}}\left(x, y_{2}\right)-\frac{\partial^{2} a}{\partial y^{2}}\left(x, y_{1}\right)\right|<\varepsilon \text { if }\left|y_{1}\right|,\left|y_{2}\right| \leq M,\left|y_{2}-y_{1}\right| \leq \delta \text {, and for a.e. } x \in \Omega .
$$

(A2) $L(\cdot, 0) \in L^{1}(\Omega)$, and for all $M>0$ there are a constant $C_{L, M}>0$ and a function $\psi_{M} \in L^{\bar{p}}(\Omega)$ such that for every $|y| \leq M$ and almost all $x \in \Omega$

$$
\left|\frac{\partial L}{\partial y}(x, y)\right| \leq \psi_{M}(x), \quad\left|\frac{\partial^{2} L}{\partial y^{2}}(x, y)\right| \leq C_{L, M} .
$$

For every $M>0$ and $\varepsilon>0$ there exists $\delta>0$, depending on $M$ and $\varepsilon$, such that

$$
\left|\frac{\partial^{2} L}{\partial y^{2}}\left(x, y_{2}\right)-\frac{\partial^{2} L}{\partial y^{2}}\left(x, y_{1}\right)\right|<\varepsilon \text { if }\left|y_{1}\right|,\left|y_{2}\right| \leq M,\left|y_{2}-y_{1}\right| \leq \delta, \text { and for a.e. } x \in \Omega .
$$

(A3) We also assume that $\Omega$ is an open and bounded domain in $\mathbb{R}^{n}, n \leq 3$, with a Lipschitz boundary $\Gamma=\bar{\Omega} \backslash \Omega$, and $A$ denotes a second-order elliptic operator of the form

$$
A y(x)=-\sum_{i, j=1}^{n} \partial_{x_{j}}\left(a_{i j}(x) \partial_{x_{i}} y(x)\right) ;
$$

the coefficients $a_{i j} \in L^{\infty}(\Omega)$ satisfy

$$
\lambda_{A}|\xi|^{2} \leq \sum_{i, j=1}^{n} a_{i j}(x) \xi_{i} \xi_{j} \quad \forall \xi \in \mathbb{R}^{n} \text { for a.e. } x \in \Omega
$$

for some $\lambda_{A}>0$. 
Hereafter, we will denote the set of admissible controls by

$$
\mathcal{U}_{\mathrm{ad}}=\left\{u \in L^{\infty}(\Omega): \alpha(x) \leq u(x) \leq \beta(x) \text { for a.a. } x \in \Omega\right\} .
$$

The reader is referred to [23, Chapter 4] for the proofs of the following theorems.

THEOREM 2.1. Under assumptions (A1) and (A3), the following statements hold:

(1) For any $u \in L^{p}(\Omega)$, with $p>n / 2$, there exists a unique (weak) solution $y_{u} \in H_{0}^{1}(\Omega)$ of the state equation (1.1). This solution satisfies $y_{u} \in Y:=$ $H_{0}^{1}(\Omega) \cap C(\bar{\Omega})$. Moreover, there exists a constant $M_{\alpha, \beta}$ such that

$$
\left\|y_{u}\right\|_{C(\bar{\Omega})}+\left\|y_{u}\right\|_{H_{0}^{1}(\Omega)} \leq M_{\alpha, \beta} \quad \forall u \in \mathcal{U}_{\mathrm{ad}}
$$

(2) The control-to-state mapping $G: L^{2}(\Omega) \longrightarrow Y$ defined by $G(u)=y_{u}$ is of class $C^{2}$. Moreover, for $v \in L^{2}(\Omega), z_{v}=G^{\prime}(u) v$ is the unique weak solution of

$$
\left\{\begin{aligned}
A z+\frac{\partial a}{\partial y}(\cdot, y) z=v & & \text { in } \Omega \\
z=0 & & \text { on } \Gamma
\end{aligned}\right.
$$

and given $v_{1}, v_{2} \in L^{2}(\Omega), w_{v_{1}, v_{2}}=G^{\prime \prime}(u)\left(v_{1}, v_{2}\right)$ is the unique weak solution of

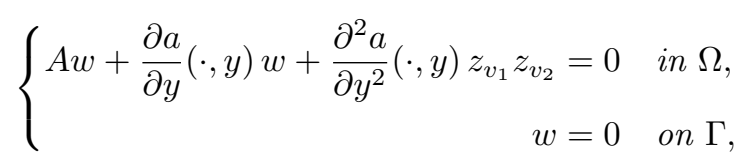

where $z_{v_{i}}=G^{\prime}(u) v_{i}, i=1,2$.

As a consequence of this theorem and assumption (A2), we get by an application of the chain rule the following result.

THEOREM 2.2. Under assumptions (A1)-(A3), the functional $J: L^{\infty}(\Omega) \longrightarrow \mathbb{R}$ is of class $C^{2}$ and the first and second derivatives are given by

$$
\begin{aligned}
J^{\prime}(u) v & =\int_{\Omega} \varphi_{u} v \mathrm{~d} x, \\
J^{\prime \prime}(u)\left(v_{1}, v_{2}\right) & =\int_{\Omega}\left[\frac{\partial^{2} L}{\partial y^{2}}\left(\cdot, y_{u}\right)-\frac{\partial^{2} a}{\partial y^{2}}\left(\cdot, y_{u}\right) \varphi_{u}\right] z_{v_{1}} z_{v_{2}} \mathrm{~d} x,
\end{aligned}
$$

where $z_{v_{i}}=G^{\prime}(u) v_{i}, i=1,2$, and $\varphi_{u} \in Y$ is the adjoint state defined as the unique weak solution of

$$
\left\{\begin{aligned}
A^{*} \varphi+\frac{\partial a}{\partial y}\left(\cdot, y_{u}\right) \varphi & =\frac{\partial L}{\partial y}\left(\cdot, y_{u}\right) & & \text { in } \Omega, \\
\varphi & =0 & & \text { on } \Gamma,
\end{aligned}\right.
$$

where $A^{*}$ is the adjoint operator of $A$.

Since the control problem (P) is not convex, we distinguish between local and global solutions. Moreover, due to the different possible norms on the control space, there are different notions of local solutions. Let us give a precise definition. 
By $B_{\varepsilon}^{p}(\bar{u})$, we denote a closed ball w.r.t. the norm in $L^{p}(\Omega)$, i.e.,

$$
B_{\varepsilon}^{p}(\bar{u}):=\left\{v \in L^{p}(\Omega):\|\bar{u}-v\|_{L^{p}(\Omega)} \leq \varepsilon\right\}
$$

for $p \in[1, \infty], \varepsilon>0$, and $\bar{u} \in L^{p}(\Omega)$.

Definition 2.3. An element $\bar{u} \in \mathcal{U}_{\mathrm{ad}}$ is said to be a solution of $(\mathrm{P})$ or, equivalently, a global minimum, if $J(\bar{u}) \leq J(u)$ for all $u \in \mathcal{U}_{\mathrm{ad}}$. We will say that $\bar{u}$ is a local minimum of $(\mathrm{P})$ in the $L^{p}(\Omega)$ sense, $p \in[1, \infty]$, if there exists a ball $B_{\varepsilon}^{p}(\bar{u})$ such that $J(\bar{u}) \leq J(u)$ for all $u \in \mathcal{U}_{\mathrm{ad}} \cap B_{\varepsilon}^{p}(\bar{u})$. The element $\bar{u}$ will be called a strict local minimum if the inequality $J(\bar{u})<J(u)$ holds for all $u \in \mathcal{U}_{\mathrm{ad}} \cap B_{\varepsilon}^{p}(\bar{u})$ with $\bar{u} \neq u$.

Since $\mathcal{U}_{\text {ad }}$ is a bounded subset of $L^{\infty}(\Omega)$, if $\bar{u}$ is a (strict) local minimum of (P) in the $L^{p}(\Omega)$ sense, for some $p \in[1, \infty)$, then $\bar{u}$ is a (strict) local minimum of $(\mathrm{P})$ in the $L^{q}(Q)$ sense for every $q \in[1, \infty]$. However, if $\bar{u}$ is a local minimum in the $L^{\infty}(\Omega)$ sense, it is not necessarily a local minimum in the $L^{p}(\Omega)$ sense for any $p \in[1, \infty)$.

The following result on first-order optimality conditions is classical; see, e.g., [23, Chapter 4].

THEOREM 2.4. The control problem (P) has at least one global minimum. Moreover, if $\bar{u}$ is a local minimum of $(\mathrm{P})$ in the $L^{p}(\Omega)$ sense, for some $p \in[1, \infty]$, then there exist a unique state $\bar{y} \in Y$ and a unique adjoint state $\bar{\varphi} \in Y$ such that the following relationships hold:

$$
\begin{array}{cclc}
A \bar{y}+a(\cdot, \bar{y})=\bar{u} & \text { in } \Omega, & \bar{y}=0 & \text { on } \Gamma, \\
A^{*} \bar{\varphi}+\frac{\partial a}{\partial y}(\cdot, \bar{y}) \bar{\varphi}=\frac{\partial L}{\partial y}(\cdot, \bar{y}) & \text { in } \Omega, & \bar{\varphi}=0 & \text { on } \Gamma, \\
\int_{\Omega} \bar{\varphi}(x)(u(x)-\bar{u}(x)) \mathrm{d} x \geq 0 & \forall u \in \mathcal{U}_{\mathrm{ad}} .
\end{array}
$$

2.2. Discussion of second-order conditions for bang-bang controls. Let now $\bar{u}$ be locally optimal for $(\mathrm{P})$ in the $L^{p}(\Omega)$ sense, $p \in[1, \infty]$. From the inequality (2.9), we deduce as usual

$$
\bar{u}(x)=\left\{\begin{array} { l l } 
{ \alpha ( x ) } & { \text { if } \overline { \varphi } ( x ) > 0 , } \\
{ \beta ( x ) } & { \text { if } \overline { \varphi } ( x ) < 0 , }
\end{array} \text { and } \overline { \varphi } ( x ) \left\{\begin{array}{ll}
\geq 0 & \text { if } \bar{u}(x)=\alpha(x), \\
\leq 0 & \text { if } \bar{u}(x)=\beta(x), \\
=0 & \text { if } \alpha(x)<\bar{u}(x)<\beta(x) .
\end{array}\right.\right.
$$

In this paper we are interested in the case where

$$
|\{x \in \Omega: \bar{\varphi}(x)=0\}|=0 .
$$

Here, $|\cdot|$ denotes the Lebesgue measure. In this case, (2.10) implies that $\bar{u}(x) \in$ $\{\alpha(x), \beta(x)\}$ for almost all points $x \in \Omega$. Hence, $\bar{u}$ is called a bang-bang control. Our goal is to give sufficient optimality conditions for local optimality of a bang-bang control satisfying the first-order optimality conditions. For nonconvex optimization problems (such as $(\mathrm{P})$ ), sufficient conditions for optimality are established in terms of the second derivative of the objective functional $J$. To this end, a cone of critical directions is defined. The natural critical cone for a point $\bar{u} \in \mathcal{U}_{\text {ad }}$ satisfying the conditions $(2.7)-(2.9)$ is given by

$$
C_{\bar{u}}:=\left\{v \in L^{2}(\Omega): J^{\prime}(\bar{u}) v=0 \text { and } v \text { satisfies }(2.12)\right\},
$$


where

$$
v(x)\left\{\begin{array}{ll}
\geq 0 & \text { if } \bar{u}(x)=\alpha(x), \\
\leq 0 & \text { if } \bar{u}(x)=\beta(x),
\end{array} \quad \text { for a.a. } x \in \Omega .\right.
$$

Note that the set of functions $v \in L^{2}(\Omega)$ satisfying (2.12) is just the tangent cone to the admissible set $\mathcal{U}_{\mathrm{ad}}$ in $L^{2}(\Omega)$. An immediate consequence of $(2.10),(2.12)$ is that $\bar{\varphi}(x) v(x) \geq 0$ for almost all $x \in \Omega$, and hence $J^{\prime}(\bar{u}) v \geq 0$. Therefore, if $v \in C_{\bar{u}}$, then the identity $\bar{\varphi}(x) v(x)=0$ a.e. in $\Omega$ holds. Thus, if $\bar{u}$ is a bang-bang control, this implies $C_{\bar{u}}=\{0\}$.

Since the admissible set $\mathcal{U}_{\text {ad }}$ is polyhedric in $L^{p}(\Omega)$, we obtain

$$
J^{\prime \prime}(\bar{u}) v^{2} \geq 0 \quad \forall v \in C_{\bar{u}}
$$

as a necessary optimality condition for local optimality in the sense of $L^{p}(\Omega), p \in$ $[1, \infty]$; see, for instance, [2, section 6.3]. Since we have $C_{\bar{u}}=\{0\}$ in the case of bangbang controls, this necessary second-order condition does not provide any additional information.

Obviously, a sufficient optimality condition cannot be posed on this trivial cone $C_{\bar{u}}$. Several authors have suggested to increase the cone of critical directions and to formulate the second-order condition on this extended cone; see $[9,15]$. Pursuing these ideas, the following extended cone was suggested in [6]: for every $\tau>0$ we define

$$
C_{\bar{u}}^{\tau}:=\left\{v \in L^{2}(\Omega): v(x)=0 \text { if }|\bar{\varphi}(x)|>\tau \text { and } v \text { satisfies }(2.12)\right\} .
$$

It is clear that $C_{\bar{u}} \subset C_{\bar{u}}^{\tau}$. Indeed, an element $v \in C_{\bar{u}}$ has to vanish at a.e. point $x$ for which $\bar{\varphi}(x) \neq 0$. However, an element $v \in C_{\bar{u}}^{\tau}$ is only required to vanish at a.e. point $x$ for which $|\bar{\varphi}(x)|>\tau$.

Now, one can pose the following sufficient second-order condition on this extended cone:

$$
\exists \kappa>0 \text { such that } J^{\prime \prime}(\bar{u}) v^{2} \geq \kappa\|v\|_{L^{2}(\Omega)}^{2} \quad \forall v \in C_{\bar{u}}^{\tau} .
$$

However, it is proved in [6] that this condition cannot be fulfilled for the problem (P). The main reason is that the control is not contained explicitly in the objective and, in particular, no $L^{2}(\Omega)$ regularization term is present in $(\mathrm{P})$. Instead of the above condition, the following one was proved to be a sufficient second-order condition for optimality:

$$
\exists \kappa>0 \text { such that } J^{\prime \prime}(\bar{u}) v^{2} \geq \kappa\left\|z_{v}\right\|_{L^{2}(\Omega)}^{2} \quad \forall v \in C_{\bar{u}}^{\tau},
$$

where $z_{v}=G^{\prime}(\bar{u}) v$ is the solution of $(2.2)$ associated to the state $\bar{y}=G(\bar{u})$. Looking at the expression for the second derivative

$$
J^{\prime \prime}(\bar{u}) v^{2}=\int_{\Omega}\left[\frac{\partial^{2} L}{\partial y^{2}}(\cdot, \bar{y})-\frac{\partial^{2} a}{\partial y^{2}}\left(\cdot, y_{u}\right) \bar{\varphi}\right] z_{v}^{2} \mathrm{~d} x,
$$

the condition (2.14) makes sense. Actually, it was proved in [6] that if $\bar{u} \in \mathcal{U}_{\text {ad }}$ satisfies (2.7)-(2.9) and (2.14), then

$$
\exists \kappa, \varepsilon>0 \text { such that } J(\bar{u})+\frac{\kappa}{2}\left\|y_{u}-\bar{y}\right\|_{L^{2}(\Omega)}^{2} \leq J(u) \quad \forall u \in \mathcal{U}_{\mathrm{ad}} \cap B_{\varepsilon}^{2}(\bar{u}) .
$$


In the present paper we perform a different approach. First, we assume that $\bar{u}$ is a bang-bang control. Additionally, we make a structural assumption on the adjoint state $\bar{\varphi}$ :

$$
\exists K>0 \text { such that }|\{x \in \Omega:|\bar{\varphi}(x)| \leq \varepsilon\}| \leq K \varepsilon \quad \forall \varepsilon>0 .
$$

This assumption and some variants of it have been made in some other mathematical contexts; see $[1,8,11,24,26]$. Property $(2.16)$ holds if $\bar{\varphi} \in C^{1}(\bar{\Omega})$ and there exists a constant $C>0$ satisfying $|\nabla \bar{\varphi}(x)| \geq C$ for all $x \in \bar{\Omega}$ such that $\bar{\varphi}(x)=0$; see [8].

In the rest of this section we make the following assumption.

(A4) We assume that $\bar{u} \in \mathcal{U}_{\text {ad }}$ and it satisfies the first-order optimality conditions (2.7)-(2.9) and the assumption (2.16).

As a consequence of (2.16) we have that (2.11) holds. Hence, $\bar{u}$ is a bang-bang control. In the next two subsections we analyze the local optimality of $\bar{u}$.

2.3. Local optimality in $L^{\infty}(\Omega)$. The goal of this subsection is to prove the following theorem.

TheOREM 2.5. Under assumptions (A1)-(A4), there exists $C_{\alpha, \beta}, \varepsilon>0$ such that

$$
J(\bar{u})+\frac{C_{\alpha, \beta}}{2}\|u-\bar{u}\|_{L^{1}(\Omega)}^{2} \leq J(u) \quad \forall u \in \mathcal{U}_{\mathrm{ad}} \cap B_{\varepsilon}^{\infty}(\bar{u}) .
$$

This theorem claims that the first-order optimality conditions along with the structural assumption (2.16) implies that $\bar{u}$ is a strict local minimum of $(\mathrm{P})$ in the $L^{\infty}(\Omega)$ sense. It is quite remarkable that no condition on the second derivative of $J$ is necessary.

However, one should bear in mind that the notion of a local minimizer in $L^{\infty}(\Omega)$ is quite weak. Indeed, if the radius of optimality $\varepsilon$ in $L^{\infty}(\Omega)$ satisfies $\varepsilon \leq \beta-\alpha$ a.e. in $\Omega$, the set $\mathcal{U}_{\text {ad }} \cap B_{\varepsilon}^{\infty}(\bar{u})$ does not contain any other bang-bang control besides $\bar{u}$. Hence, (2.17) does not allow one to compare $\bar{u}$ with other bang-bang controls.

In order to prove this theorem we first establish the following lemma.

Lemma 2.6. There exists a constant $C_{\alpha, \beta}>0$ such that

$$
\left\|\varphi_{u}-\bar{\varphi}\right\|_{C(\bar{\Omega})} \leq C_{\alpha, \beta}\|u-\bar{u}\|_{L^{1}(\Omega)} \quad \forall u \in \mathcal{U}_{\mathrm{ad}},
$$

where $\varphi_{u}$ and $\bar{\varphi}$ are the solutions of (2.6) and (2.8), respectively.

Proof. Let $u \in \mathcal{U}_{\mathrm{ad}}$ be given, and we denote the associated state with $y_{u}$.

First, we show a measurable mean value theorem for the Carathéodory function $a$. We consider the Carathéodory function $F: \Omega \times[0,1] \longrightarrow \mathbb{R}$ given by

$$
F(x, t)=\left|a\left(x, y_{u}(x)\right)-a(x, \bar{y}(x))-\frac{\partial a}{\partial y}\left(x, \bar{y}(x)+t\left[y_{u}(x)-\bar{y}(x)\right]\right)\left(y_{u}(x)-\bar{y}(x)\right)\right|,
$$

and [10, Proposition VIII.1.1] implies that $F$ is a normal integrand. Now, from the scalar mean value theorem and [10, Theorem VIII.1.2] we deduce the existence of a measurable function $\theta: \Omega \longrightarrow[0,1]$ such that

$$
F(x, \theta(x))=\min _{t \in[0,1]} F(x, t)=0 \quad \forall x \in \Omega .
$$

Then, we subtract the state equations satisfied by $y_{u}$ and $\bar{y}$ and obtain

$$
\begin{aligned}
A\left(y_{u}-\bar{y}\right)+\frac{\partial a}{\partial y}\left(\cdot, y_{\theta}\right)\left(y_{u}-\bar{y}\right) & =u-\bar{u} & & \text { in } \Omega, \\
y_{u}-\bar{y} & =0 & & \text { on } \Gamma,
\end{aligned}
$$


where $y_{\theta}=\bar{y}+\theta\left(y_{u}-\bar{y}\right)$ and $\theta: \Omega \rightarrow[0,1]$ is measurable. The next step is to bound $\left\|y_{u}-\bar{y}\right\|_{L^{2}(\Omega)}$ by $\|u-\bar{u}\|_{L^{1}(\Omega)}$. This is achieved by using results for PDEs with measures on the right-hand side. We note that (A1) and (2.1) imply that $0 \leq$ $\partial a / \partial y\left(\cdot, y_{\theta}\right) \in L^{\infty}(\Omega)$. Thus, we can employ the technique of [16, Theorem 2.12] (see also the classical work [22, Théorème 9.1]), and we obtain the existence of a constant $D_{\alpha, \beta}$ such that

$$
\left\|y_{u}-\bar{y}\right\|_{L^{2}(\Omega)} \leq D_{\alpha, \beta}\|u-\bar{u}\|_{L^{1}(\Omega)} \quad \forall u \in \mathcal{U}_{\mathrm{ad}} .
$$

Now, subtracting (2.6) and (2.8) we obtain

$$
\left\{\begin{array}{rlrl}
A^{*}\left(\varphi_{u}-\bar{\varphi}\right)+\frac{\partial a}{\partial y}(\cdot, \bar{y})\left(\varphi_{u}-\bar{\varphi}\right) & =\frac{\partial L}{\partial y}\left(\cdot, y_{u}\right)-\frac{\partial L}{\partial y}(\cdot, \bar{y}) & \\
& -\left[\frac{\partial a}{\partial y}\left(\cdot, y_{u}\right)-\frac{\partial a}{\partial y}(\cdot, \bar{y})\right] \varphi_{u} & & \text { in } \Omega, \\
\varphi_{u}-\bar{\varphi} & =0 & & \text { on } \Gamma,
\end{array}\right.
$$

Hence, $\left\|\varphi_{u}-\bar{\varphi}\right\|_{C(\bar{\Omega})}$ is estimated by the $L^{2}(\Omega)$ norm of the right-hand side of the equation. To this end we first observe that (2.1) implies that the solution of (2.6) satisfies $\left\|\varphi_{u}\right\|_{C(\bar{\Omega})} \leq C$ for some constant $C$ and for all $u \in \mathcal{U}_{\text {ad }}$. Moreover, assumptions (A1) and (A2) along with the mean value theorem imply

$$
\begin{aligned}
\left|\frac{\partial L}{\partial y}\left(x, y_{u}(x)\right)-\frac{\partial L}{\partial y}(x, \bar{y}(x))\right|+ & \left|\frac{\partial a}{\partial y}\left(x, y_{u}(x)\right)-\frac{\partial a}{\partial y}(x, \bar{y}(x))\right| \\
& \leq\left[C_{L, M_{\alpha, \beta}}+C_{a, M_{\alpha, \beta}}\right]\left|y_{u}(x)-\bar{y}(x)\right|,
\end{aligned}
$$

which yields with $(2.19)$

$$
\left\|\frac{\partial L}{\partial y}\left(\cdot, y_{u}\right)-\frac{\partial L}{\partial y}(\cdot, \bar{y})+\left(\frac{\partial a}{\partial y}\left(\cdot, y_{u}\right)-\frac{\partial a}{\partial y}(\cdot, \bar{y})\right) \phi_{u}\right\|_{L^{2}(\Omega)} \leq D_{\alpha, \beta}\|u-\bar{u}\|_{L^{1}(\Omega)} .
$$

From this inequality (2.18) follows.

Now, we are in position to prove Theorem 2.5.

Proof of Theorem 2.5. Let us set

$$
\varepsilon:=\frac{1}{8 K C_{\alpha, \beta}},
$$

where $K$ and $C_{\alpha, \beta}$ satisfy the structural assumption (2.16) and the Lipschitz estimate (2.18), respectively. Now, we take $u \in \mathcal{U}_{\text {ad }} \cap B_{\varepsilon}^{\infty}(\bar{u})$. With the mean value theorem we get

$$
J(u)-J(\bar{u})=J^{\prime}\left(u_{\theta}\right)(u-\bar{u}) \quad \text { with } u_{\theta}=\bar{u}+\theta(u-\bar{u}) \text { and } 0 \leq \theta \leq 1 .
$$

Let $\varphi_{\theta}$ denote the adjoint state associated with $u_{\theta}$, and set

$$
E:=\left\{x \in \Omega:\left|\varphi_{\theta}(x)\right| \geq C_{\alpha, \beta}\|u-\bar{u}\|_{L^{1}(\Omega)}\right\} \text { and } F:=\Omega \backslash E .
$$

From (2.10) and the fact that $\alpha \leq u \leq \beta$ a.e. in $\Omega$, we know that $\bar{\varphi}(x)(u(x)-\bar{u}(x)) \geq 0$ for almost all $x \in \Omega$. Moreover, $\operatorname{sign} \varphi_{\theta}(x)=\operatorname{sign} \bar{\varphi}(x)$ holds for all $x \in E$. Let us 
check this claim. If $x \in E$ and $\varphi_{\theta}(x)>0$, then $\varphi_{\theta}(x)>C_{a, \beta}\|u-\bar{u}\|_{L^{1}(\Omega)}$. Then, (2.18) implies

$$
\bar{\varphi}(x)=\varphi_{\theta}(x)+\left[\bar{\varphi}(x)-\varphi_{\theta}(x)\right] \geq \varphi_{\theta}(x)-C_{a, \beta}\|u-\bar{u}\|_{L^{1}(\Omega)}>0 .
$$

Analogously, we proceed if $\varphi_{\theta}(x)<0$ for some $x \in E$.

Combining these two observations we infer

$$
\varphi_{\theta}(x)(u(x)-\bar{u}(x)) \geq 0 \quad \forall x \in E .
$$

Let us prove that

$$
|F| \leq 2 K C_{\alpha, \beta}\|u-\bar{u}\|_{L^{1}(\Omega)} .
$$

To this end, we first define

$$
\bar{F}:=\left\{x \in \Omega:|\bar{\varphi}(x)| \leq 2 C_{\alpha, \beta}\|u-\bar{u}\|_{L^{1}(\Omega)}\right\} .
$$

From the definition of $F$ and (2.18) we obtain

$$
x \in F \Rightarrow|\bar{\varphi}(x)| \leq\left|\varphi_{\theta}(x)\right|+\left|\bar{\varphi}(x)-\varphi_{\theta}(x)\right|<2 C_{\alpha, \beta}\|u-\bar{u}\|_{L^{1}(\Omega)} \Rightarrow x \in \bar{F} .
$$

Hence, (2.23) follows from the inclusion $F \subset \bar{F}$ and the structural assumption (2.16). Now, using (2.22) and (2.23) we get

$$
\begin{aligned}
\| u & -\bar{u}\left\|_{L^{1}(\Omega)}^{2}=\right\| u-\bar{u} \|_{L^{1}(\Omega)}\left\{\int_{E}|u-\bar{u}| \mathrm{d} x+\int_{F}|u-\bar{u}| \mathrm{d} x\right\} \\
& \leq \frac{1}{C_{\alpha, \beta}} \int_{E} \varphi_{\theta}(u-\bar{u}) \mathrm{d} x+\|u-\bar{u}\|_{L^{1}(\Omega)} \int_{F}|u-\bar{u}| \mathrm{d} x \\
& =\frac{1}{C_{\alpha, \beta}} J^{\prime}\left(u_{\theta}\right)(u-\bar{u})-\frac{1}{C_{\alpha, \beta}} \int_{F} \varphi_{\theta}(u-\bar{u}) \mathrm{d} x+\|u-\bar{u}\|_{L^{1}(\Omega)} \int_{F}|u-\bar{u}| \mathrm{d} x \\
& \leq \frac{1}{C_{\alpha, \beta}} J^{\prime}\left(u_{\theta}\right)(u-\bar{u})+2\|u-\bar{u}\|_{L^{1}(\Omega)} \int_{F}|u-\bar{u}| \mathrm{d} x \\
& \leq \frac{1}{C_{\alpha, \beta}} J^{\prime}\left(u_{\theta}\right)(u-\bar{u})+2\|u-\bar{u}\|_{L^{1}(\Omega)}\|u-\bar{u}\|_{L^{\infty}(\Omega)}|F| \\
& \leq \frac{1}{C_{\alpha, \beta}} J^{\prime}\left(u_{\theta}\right)(u-\bar{u})+4 K C_{\alpha, \beta}\|u-\bar{u}\|_{L^{\infty}(\Omega)}\|u-\bar{u}\|_{L^{1}(\Omega)}^{2} .
\end{aligned}
$$

From here and (2.21) we deduce

$$
C_{\alpha, \beta}\left\{1-4 K C_{\alpha, \beta}\|u-\bar{u}\|_{L^{\infty}(\Omega)}\right\}\|u-\bar{u}\|_{L^{1}(\Omega)}^{2} \leq J(u)-J(\bar{u}) .
$$

Finally, from (2.20) and the fact that $\|u-\bar{u}\|_{L^{\infty}(\Omega)}<\varepsilon$ we conclude (2.17).

2.4. Local optimality in $L^{\mathbf{1}}(\Omega)$. In this section we provide a condition for $\bar{u}$ to be a strict local minimum of $(\mathrm{P})$ in the sense of $L^{1}(\Omega)$, and consequently in the $L^{p}(\Omega)$ sense for every $p \in[1, \infty]$. This optimality condition is based on a weak second-order condition. Before establishing the main result, let us prove the following proposition.

Proposition 2.7. Let us assume that (A1)-(A4) hold; then there exists $\kappa>0$ such that

$$
J^{\prime}(\bar{u})(u-\bar{u}) \geq \kappa\|u-\bar{u}\|_{L^{1}(\Omega)}^{2} \quad \forall u \in \mathcal{U}_{\mathrm{ad}}
$$


Note that $\mathcal{U}_{\text {ad }}$ is a bounded subset of $L^{\infty}(\Omega)$. In particular, $\mathcal{U}_{\text {ad }}$ is not a cone. The proof below will reveal that $\kappa$ can be chosen proportional to $\|u-\bar{u}\|_{L^{\infty}(\Omega)}^{-1}$, which is bounded below by $\|\beta-\alpha\|_{L^{\infty}(\Omega)}^{-1}$, and the scaling of $u-\bar{u}$ in (2.24) is indeed correct.

Proof of Proposition 2.7. Given $u \in \mathcal{U}_{\text {ad }}$, we set

$$
\varepsilon:=\left(2\|\beta-\alpha\|_{L^{\infty}(\Omega)} K\right)^{-1}\|u-\bar{u}\|_{L^{1}(\Omega)} \quad \text { and } \quad E_{\varepsilon}:=\{x \in \Omega:|\bar{\varphi}(x)| \geq \varepsilon\} .
$$

Then, we have

$$
\begin{aligned}
J^{\prime}(\bar{u})(u-\bar{u}) & =\int_{\Omega}\left|\bar{\varphi}\left\|u-\bar{u}\left|\mathrm{~d} x \geq \int_{E_{\varepsilon}}\right| \bar{\varphi}\right\| u-\bar{u}\right| \mathrm{d} x \\
& \geq \varepsilon\|u-\bar{u}\|_{L^{1}\left(E_{\varepsilon}\right)}=\varepsilon\left(\|u-\bar{u}\|_{L^{1}(\Omega)}-\|u-\bar{u}\|_{L^{1}\left(\Omega \backslash E_{\varepsilon}\right)}\right) .
\end{aligned}
$$

Since $\left|\Omega \backslash E_{\varepsilon}\right| \leq K \varepsilon$ due to the structural assumption (2.16), we find

$$
\|u-\bar{u}\|_{L^{1}\left(\Omega \backslash E_{\varepsilon}\right)} \leq\|\beta-\alpha\|_{L^{\infty}(\Omega)} K \varepsilon .
$$

Hence we get

$$
\begin{aligned}
J^{\prime}(\bar{u})(u-\bar{u}) & \geq \varepsilon\left(\|u-\bar{u}\|_{L^{1}(\Omega)}-\|u-\bar{u}\|_{L^{1}\left(\Omega \backslash E_{\varepsilon}\right)}\right) \\
& \geq \varepsilon\left(\|u-\bar{u}\|_{L^{1}(\Omega)}-\|\beta-\alpha\|_{L^{\infty}(\Omega)} K \varepsilon\right)=\kappa\|u-\bar{u}\|_{L^{1}(\Omega)}^{2}
\end{aligned}
$$

with $\kappa=\left(4\|\beta-\alpha\|_{L^{\infty}(\Omega)} K\right)^{-1}$.

Now, we formulate a second-order condition on the extended cone $C_{\bar{u}}^{\tau}$, defined in (2.13).

Here, we allow for some negative curvature of the second derivative on critical directions. Hence, the assumption on $J^{\prime \prime}(\bar{u})$ is very weak when compared to standard second-order conditions, which require positive definiteness of $J^{\prime \prime}(\bar{u})$. This weakening is possible due to the structural assumption (2.16) and its consequence, Proposition 2.7, which gives second-order growth from a first-order expression.

THEOREM 2.8. Suppose that assumptions (A1)-(A4) hold. Further, we assume that

$$
\exists \tau>0, \exists \kappa^{\prime}<\kappa: \quad J^{\prime \prime}(\bar{u}) v^{2} \geq-\kappa^{\prime}\|v\|_{L^{1}(\Omega)}^{2} \quad \forall v \in C_{\bar{u}}^{\tau},
$$

where the constant $\kappa$ is given in Proposition 2.7. Then, there exists $\varepsilon>0$ such that

$$
J(\bar{u})+\frac{\kappa-\kappa^{\prime}}{8}\|u-\bar{u}\|_{L^{1}(\Omega)}^{2} \leq J(u) \quad \forall u \in \mathcal{U}_{\mathrm{ad}} \cap B_{\varepsilon}^{1}(\bar{u}) .
$$

Proof. Given $\rho>0$, there exists $\varepsilon_{\rho}>0$ such that for every $u \in \mathcal{U}_{\text {ad }}$ with $\| u-$ $\bar{u} \|_{L^{2}(\Omega)} \leq \varepsilon_{\rho}$ we have

$$
\left|\left[J^{\prime \prime}(u)-J^{\prime \prime}(\bar{u})\right] v^{2}\right| \leq \rho\left\|z_{v}\right\|_{L^{2}(\Omega)}^{2} \quad \forall v \in L^{2}(\Omega),
$$

where $z_{v}=G^{\prime}(\bar{u}) v$; see $\left[6\right.$, Lemma 2.7]. Since $\|u-\bar{u}\|_{L^{2}(\Omega)} \leq\|\beta-\alpha\|_{L^{\infty}(\Omega)}^{1 / 2}\|u-\bar{u}\|_{L^{1}(\Omega)}^{1 / 2}$ and $\left\|z_{v}\right\|_{L^{2}(\Omega)} \leq C_{1}\|v\|_{L^{1}(\Omega)}$ (which follows from (2.2)), we deduce the existence of $\varepsilon_{0}>0$ such that for every $u \in \mathcal{U}_{\text {ad }}$ with $\|u-\bar{u}\|_{L^{1}(\Omega)}<\varepsilon_{0}$

$$
\left|\left[J^{\prime \prime}(u)-J^{\prime \prime}(\bar{u})\right] v^{2}\right| \leq \frac{\kappa-\kappa^{\prime}}{2}\|v\|_{L^{1}(\Omega)}^{2} \quad \forall v \in L^{2}(\Omega) .
$$


On the other hand, from assumptions (A1) and (A2), (2.1), and the expression (2.5) we get

$$
\left|J^{\prime \prime}(\bar{u})\left(v_{1}, v_{2}\right)\right| \leq C_{2}\left\|z_{v_{1}}\right\|_{L^{2}(\Omega)}\left\|z_{v_{2}}\right\|_{L^{2}(\Omega)} \leq C\left\|v_{1}\right\|_{L^{1}(\Omega)}\left\|v_{2}\right\|_{L^{1}(\Omega)}
$$

with $C=C_{1}^{2} C_{2}$. We define the set $\Omega_{\tau}=\{x \in \Omega:|\bar{\varphi}(x)|>\tau\}$. Let us observe that (2.10) and (2.24) imply that

$$
\begin{aligned}
J^{\prime}(\bar{u})(u-\bar{u}) & \geq \frac{1}{2} J^{\prime}(\bar{u})(u-\bar{u})+\frac{1}{2} \int_{\Omega_{\tau}}|\bar{\varphi}(x) \| u(x)-\bar{u}(x)| \mathrm{d} x \\
& \geq \frac{\kappa}{2}\|u-\bar{u}\|_{L^{1}(\Omega)}^{2}+\frac{\tau}{2}\|u-\bar{u}\|_{L^{1}\left(\Omega_{\tau}\right) .}
\end{aligned}
$$

Now, given $u \in \mathcal{U}_{\text {ad }}$ with $\|u-\bar{u}\|_{L^{1}(\Omega)}<\varepsilon_{0}$, we define

$$
u_{1}(x):=\left\{\begin{array}{ll}
\bar{u}(x) & \text { if } x \in \Omega_{\tau}, \\
u(x) & \text { otherwise, }
\end{array} \text { and } u_{2}(x):= \begin{cases}u(x)-\bar{u}(x) & \text { if } x \in \Omega_{\tau}, \\
0 & \text { otherwise. }\end{cases}\right.
$$

Then we have that $u=u_{1}+u_{2},\left(u_{1}-\bar{u}\right) \in C_{\bar{u}}^{\tau}$, and $\left|u_{1}-\bar{u}\right| \leq|u-\bar{u}|$. By making a Taylor expansion and using (2.25), (2.27), (2.28), (2.29), and Young's inequality we obtain for some $u_{\theta}=\bar{u}+\theta(u-\bar{u})$ with $0 \leq \theta \leq 1$

$$
\begin{aligned}
J(u) & -J(\bar{u})=J^{\prime}(\bar{u})(u-\bar{u})+\frac{1}{2} J^{\prime \prime}\left(u_{\theta}\right)(u-\bar{u})^{2} \\
\geq & \frac{\kappa}{2}\|u-\bar{u}\|_{L^{1}(\Omega)}^{2}+\frac{\tau}{2}\|u-\bar{u}\|_{L^{1}\left(\Omega_{\tau}\right)}+\frac{1}{2} J^{\prime \prime}(\bar{u})(u-\bar{u})^{2}-\frac{\kappa-\kappa^{\prime}}{4}\|u-\bar{u}\|_{L^{1}(\Omega)}^{2} \\
= & \frac{\kappa+\kappa^{\prime}}{4}\|u-\bar{u}\|_{L^{1}(\Omega)}^{2}+\frac{\tau}{2}\|u-\bar{u}\|_{L^{1}\left(\Omega_{\tau}\right)}+\frac{1}{2} J^{\prime \prime}(\bar{u})\left(u_{1}-\bar{u}\right)^{2}+\frac{1}{2} J^{\prime \prime}(\bar{u}) u_{2}^{2} \\
& +J^{\prime \prime}(\bar{u})\left(u_{1}-\bar{u}, u_{2}\right) \\
\geq & \frac{\kappa+\kappa^{\prime}}{4}\|u-\bar{u}\|_{L^{1}(\Omega)}^{2}+\frac{\tau}{2}\|u-\bar{u}\|_{L^{1}\left(\Omega_{\tau}\right)}-\frac{\kappa^{\prime}}{2}\left\|u_{1}-\bar{u}\right\|_{L^{1}(\Omega)}^{2} \\
& -\frac{C}{2}\left\|u_{2}\right\|_{L^{1}(\Omega)}^{2}-C\left\|u_{1}-\bar{u}\right\|_{L^{1}(\Omega)}\left\|u_{2}\right\|_{L^{1}(\Omega)} \\
\geq & \frac{\kappa-\kappa^{\prime}}{4}\|u-\bar{u}\|_{L^{1}(\Omega)}^{2}+\frac{\tau}{2}\|u-\bar{u}\|_{L^{1}\left(\Omega_{\tau}\right)} \\
& -\frac{C}{2}\|u-\bar{u}\|_{L^{1}\left(\Omega_{\tau}\right)}^{2}-C\|u-\bar{u}\|_{L^{1}(\Omega)}\|u-\bar{u}\|_{L^{1}\left(\Omega_{\tau}\right)} \\
\geq & \frac{\kappa-\kappa^{\prime}}{8}\|u-\bar{u}\|_{L^{1}(\Omega)}^{2}+\frac{\tau}{2}\|u-\bar{u}\|_{L^{1}\left(\Omega_{\tau}\right)}-\left(\frac{C}{2}+\frac{2 C^{2}}{\kappa-\kappa^{\prime}}\right)\|u-\bar{u}\|_{L^{1}\left(\Omega_{\tau}\right)}^{2} .
\end{aligned}
$$

Finally, we take

$$
\varepsilon=\min \left\{\varepsilon_{0},\left(\frac{C}{2}+\frac{2 C^{2}}{\kappa-\kappa^{\prime}}\right)^{-1} \frac{\tau}{2}\right\} .
$$

Then (2.26) follows from the above inequality just by noting that the sum of the last two terms is nonnegative if $\|u-\bar{u}\|_{L^{1}(\Omega)}<\varepsilon$.

Remark 2.9. Let us observe that in the proof of Theorem 2.8 the assumption (2.25) was used to deduce that

$$
J^{\prime \prime}(\bar{u})\left(u_{1}-\bar{u}\right)^{2} \geq-\kappa^{\prime}\left\|u_{1}-\bar{u}\right\|_{L^{1}(\Omega)}^{2} \geq-\kappa^{\prime}\|u-\bar{u}\|_{L^{1}(\Omega)}^{2} .
$$


The reader could think that the assumption (2.25) is too strong and it could be relaxed by assuming that

$$
\begin{gathered}
J^{\prime \prime}(\bar{u})(u-\bar{u})^{2} \geq-\kappa^{\prime}\|u-\bar{u}\|_{L^{1}(\Omega)}^{2} \quad \forall u \in \mathcal{U}_{\mathrm{ad}} \text { satisfying } \\
\|u-\bar{u}\|_{L^{1}(\Omega)}<\varepsilon \text { and } u(x)=\bar{u}(x) \text { if }|\bar{\varphi}(x)|>\tau .
\end{gathered}
$$

However, it is not difficult to prove that this assumption is equivalent to (2.25).

2.5. Second-order condition involving measures concentrated on the set $\{\bar{\varphi}(x)=0\}$. Let us now discuss a second-order condition that involves measures that are concentrated on the set of discontinuities of $\bar{u}$. Let us denote

$$
Z_{\bar{\varphi}}:=\{x \in \Omega: \bar{\varphi}(x)=0\} .
$$

On this set we consider the space $\mathcal{M}\left(Z_{\bar{\varphi}}\right)$ formed by the real-valued and regular Borel measures in $Z_{\bar{\varphi}}$. Looking for an extension of the results well established for finitedimensional optimization problems, we should check the second derivative $J^{\prime \prime}(\bar{u})$ on elements defined on the set of points where the derivative $J^{\prime}(\bar{u})=\bar{\varphi}$ vanishes. This set $Z_{\bar{\varphi}}$ has a zero Lebesgue measure, and hence it is quite natural to replace functions by Borel measures belonging to the space $\mathcal{M}\left(Z_{\bar{\varphi}}\right)$. Notice that $Z_{\bar{\varphi}}$ can be a set formed by a finite amount of points, or a line in dimension 2 , or a surface in dimension 3 .

According to the Riesz representation theorem we have $\mathcal{M}\left(Z_{\bar{\varphi}}\right)=C_{0}\left(Z_{\bar{\varphi}}\right)^{*}$; see [21, Theorem 6.19]. We also denote by $\mathcal{M}(\Omega)=C_{0}(\Omega)^{*}$ the space of real-valued and regular Borel measures in $\Omega$. $\mathcal{M}\left(Z_{\bar{\varphi}}\right)$ can be considered as a subspace of $\mathcal{M}(\Omega)$. Indeed it is enough to extend any measure $\mu \in \mathcal{M}\left(Z_{\bar{\varphi}}\right)$ by zero outside $Z_{\bar{\varphi}}$. For every measure in $\mathcal{M}(\Omega)$, and hence also for all $\mu \in \mathcal{M}\left(Z_{\bar{\varphi}}\right)$, the linearized state equation (2.2) with $v=\mu$ has a unique solution $z_{\mu} \in W_{0}^{1, p}(\Omega)$ for every $1 \leq p<\frac{n}{n-1}$ and

$$
\left\|z_{\mu}\right\|_{W_{0}^{1, p}(\Omega)} \leq C_{p}\|\mu\|_{\mathcal{M}\left(Z_{\bar{\varphi}}\right)}
$$

see [16, Theorem 2.12] and [22, Théorème 9.1]. From the embedding $W_{0}^{1, p}(\Omega) \subset L^{2}(\Omega)$ for $p \geq \frac{2 n}{n+2}$ we infer that $\left\|z_{\mu}\right\|_{L^{2}(\Omega)} \leq C_{p, 2}\|\mu\|_{\mathcal{M}\left(Z_{\bar{\varphi}}\right)}$. Hence, from the expression (2.5) and assumptions (A1) and (A2) we get that the bilinear form $J^{\prime \prime}(\bar{u})$ can be extended from $L^{2}(\Omega)$ to a continuous bilinear form on $\mathcal{M}\left(Z_{\bar{\varphi}}\right)$. In particular we have

$$
J^{\prime \prime}(\bar{u}) \mu^{2}=\int_{\Omega}\left[\frac{\partial^{2} L}{\partial y^{2}}(x, \bar{y})-\frac{\partial^{2} a}{\partial y^{2}}(x, \bar{y}) \varphi_{u}\right] z_{\mu}^{2} \mathrm{~d} x \quad \forall \mu \in \mathcal{M}\left(Z_{\bar{\varphi}}\right) .
$$

Finally, we mention a continuity property of $J^{\prime \prime}(\bar{u})$. As in [16, Lemma 2.15], we can show that $v_{k} \stackrel{*}{\rightarrow} \mu$ in $\mathcal{M}\left(Z_{\bar{\varphi}}\right)$ implies $z_{v_{k}} \rightarrow z_{\mu}$ in $L^{2}(\Omega)$, and, consequently, $J^{\prime \prime}(\bar{u}) v_{k}^{2} \rightarrow J^{\prime \prime}(\bar{u}) \mu^{2}$.

It is natural to impose a second-order condition on the set where the derivative of the functional vanishes, namely on the set $Z_{\bar{\varphi}}$. We also have to take into account the set where the control constraint is active. To this end we should consider a cone in $\mathcal{M}\left(Z_{\bar{\varphi}}\right)$ that includes a condition analogous to (2.12) in its definition. Thus we define the sets

$$
\begin{aligned}
& Z_{\bar{\varphi}}^{+}=\left\{x \in Z_{\bar{\varphi}}: \exists \rho_{x}>0 \text { such that } B_{\rho_{x}}(x) \subset \Omega \text { and } \bar{\varphi}(\xi) \geq 0 \forall \xi \in B_{\rho_{x}}(x)\right\}, \\
& Z_{\bar{\varphi}}^{-}=\left\{x \in Z_{\bar{\varphi}}: \exists \rho_{x}>0 \text { such that } B_{\rho_{x}}(x) \subset \Omega \text { and } \bar{\varphi}(\xi) \leq 0 \forall \xi \in B_{\rho_{x}}(x)\right\} .
\end{aligned}
$$


Here, $B_{\rho}(x)$ denotes an open ball with radius $\rho>0$ centered at $x$. In some cases one or both of these sets can be empty. Now we define the cone

$$
\mathcal{T}_{\bar{u}}=\left\{\mu \in \mathcal{M}\left(Z_{\bar{\varphi}}\right): \mu^{+}\left(Z_{\bar{\varphi}}^{-}\right)=\mu^{-}\left(Z_{\bar{\varphi}}^{+}\right)=0\right\},
$$

where $\mu=\mu^{+}-\mu^{-}$denotes the Jordan decomposition of $\mu$.

In what follows, we analyze the second-order condition

$$
\exists \kappa^{\prime}<\kappa: \quad J^{\prime \prime}(\bar{u}) \mu^{2} \geq-\kappa^{\prime}\|\mu\|_{\mathcal{M}\left(Z_{\bar{\varphi}}\right)}^{2} \forall \mu \in \mathcal{T}_{\bar{u}} .
$$

This condition seems to be weaker than (2.25), which was formulated on the extended cone $C_{\bar{u}}^{\tau}$. However, this is not the case, as we prove below. In fact, we will show that both conditions are equivalent.

The key to this result will be that the cone $\mathcal{T}_{\bar{u}}$ is a certain limit of $C_{\bar{u}}^{\tau}$ for $\tau \searrow 0$. To this end, we denote

$$
\begin{aligned}
\mathcal{S}_{\bar{u}}=\left\{\mu \in \mathcal{M}(\Omega): \exists\left\{\tau_{k}\right\}_{k=1}^{\infty},\left\{v_{k}\right\}_{k=1}^{\infty} \text { with } \tau_{k} \searrow 0, v_{k} \in C_{\bar{u}}^{\tau_{k}},\right. \\
\text { and } \left.v_{k} \stackrel{*}{\rightarrow} \mu \text { in } \mathcal{M}(\Omega)\right\} .
\end{aligned}
$$

This set contains all measures that can be approximated by elements of $C_{\bar{u}}^{\tau}$ for $\tau \searrow 0$. That is, $\mathcal{S}_{\bar{u}}$ is the Painlevé-Kuratowski upper limit $\lim \sup _{\tau \searrow 0} C_{\bar{u}}^{\tau}$ w.r.t. weak-star convergence in $\mathcal{M}(\Omega)$. Let us note that if we take the upper limit w.r.t. strong convergence in $L^{2}(\Omega)$, we obtain the trivial cone $\{0\}$. In order to prove that $(2.25)$ implies (2.30) we will need the smaller set

$$
\begin{aligned}
\hat{\mathcal{S}}_{\bar{u}}=\left\{\mu \in \mathcal{M}(\Omega): \exists\left\{\tau_{k}\right\}_{k=1}^{\infty},\right. & \left\{v_{k}\right\}_{k=1}^{\infty} \text { with } \tau_{k} \searrow 0, v_{k} \in C_{\bar{u}_{k}}^{\tau_{k}}, \\
v_{k} & \left.\stackrel{*}{*} \mu \text { in } \mathcal{M}(\Omega), \text { and }\left\|v_{k}\right\|_{L^{1}(\Omega)} \rightarrow\|\mu\|_{\mathcal{M}(\Omega)}\right\} .
\end{aligned}
$$

Clearly, it holds that $\hat{\mathcal{S}}_{\bar{u}} \subset \mathcal{S}_{\bar{u}}$. In the proof of equivalence, the inclusions $\mathcal{S}_{\bar{u}} \subset \mathcal{T}_{\bar{u}} \subset$ $\hat{\mathcal{S}}_{\bar{u}}$ will be essential, which imply $\mathcal{T}_{\bar{u}}=\mathcal{S}_{\bar{u}}=\hat{\mathcal{S}}_{\bar{u}}$. At first, we have the following result on the sets $Z_{\bar{\varphi}}^{+}$and $Z_{\bar{\varphi}}^{-}$.

Lemma 2.10. Suppose that (2.11) holds. Then the sets $Z_{\bar{\varphi}}^{+}$and $Z_{\bar{\varphi}}^{-}$are relatively open and disjoint subsets of $Z_{\bar{\varphi}}$.

Proof. Let us prove that $Z_{\bar{\varphi}}^{+}$is a relatively open set in $Z_{\bar{\varphi}}$; the proof for $Z_{\bar{\varphi}}^{-}$is analogous. Given $\bar{x} \in Z_{\bar{\varphi}}^{+}$there exists $\bar{\rho}>0$ such that $\bar{\varphi}(\xi) \geq 0$ for all $\xi \in B_{\bar{\rho}}(\bar{x})$. It is enough to notice that $B_{\bar{\rho}}(\bar{x}) \cap Z_{\bar{\varphi}} \subset Z_{\bar{\varphi}}^{+}$. Indeed, for any element $x \in B_{\bar{\rho}}(\bar{x}) \cap Z_{\bar{\varphi}}$ we know the existence of $\rho_{x}>0$ such that $B_{\rho_{x}}(x) \subset B_{\bar{\rho}}(\bar{x})$, and hence $\bar{\varphi}(\xi) \geq 0$ for all $\xi \in B_{\rho_{x}}(x)$. Therefore, we have that $x \in Z_{\bar{\varphi}}^{+}$. Now we prove by contradiction that $Z_{\bar{\varphi}}^{+} \cap Z_{\bar{\varphi}}^{-}=\emptyset$. Let us assume that $\bar{x}$ belongs to the intersection of both sets. Then, there exist $\rho_{1}>0$ and $\rho_{2}>0$ such that

$$
\bar{\varphi}(\xi) \geq 0 \quad \forall \xi \in B_{\rho_{1}}(\bar{x}) \text { and } \bar{\varphi}(\xi) \leq 0 \quad \forall \xi \in B_{\rho_{2}}(\bar{x}) .
$$

Then, $\bar{\varphi}(\xi)=0$ for all $\xi \in B_{\rho}(\bar{x})$ holds with $\rho=\min \left\{\rho_{1}, \rho_{2}\right\}$. This contradicts (2.11).

With the help of this result, the extension by zero of functions from $C_{0}\left(Z_{\bar{\varphi}}^{+}\right)$and $C_{0}\left(Z_{\bar{\varphi}}^{-}\right)$to $Z_{\bar{\varphi}}$ belongs to $C_{0}\left(Z_{\bar{\varphi}}\right)$. This allows us to prove the inclusion $\mathcal{S}_{\bar{u}} \subset \mathcal{T}_{\bar{u}}$.

Lemma 2.11. Suppose that (2.11) holds. Then it holds that $\mathcal{S}_{\bar{u}} \subset \mathcal{T}_{\bar{u}}$. 
Proof. First we prove that $\mathcal{S}_{\bar{u}} \subset \mathcal{M}\left(Z_{\bar{\varphi}}\right)$. To this end we take an element $\mu \in \mathcal{S}_{\bar{u}}$ and a sequence $\left\{v_{k}\right\}_{k=1}^{\infty}$ with $v_{k} \in C_{\bar{u}}^{\tau_{k}}$ for every $k \geq 1$ and $\tau_{k} \searrow 0$, and such that $v_{k} \stackrel{*}{\rightarrow} \mu$. Now, for an arbitrary open set $V$ with $V \subset \bar{V} \subset \Omega \backslash \bar{Z}_{\bar{\varphi}}$, we deduce the existence of $\varepsilon>0$ such that $|\bar{\varphi}(x)|>\varepsilon$ for every $x \in V$. Then the convergence $\tau_{k} \searrow 0$ implies that $v_{k}=0$ in $V$ for all $k$ big enough, namely for $\tau_{k}<\varepsilon$. From here we infer for $z \in C_{0}(V)$ extended by zero to $\Omega$ that

$$
\int_{V} z(x) \mathrm{d} \mu(x)=\int_{\Omega} z(x) \mathrm{d} \mu(x)=\lim _{k \rightarrow \infty} \int_{\Omega} z(x) v_{k}(x) \mathrm{d} x=\lim _{k \rightarrow \infty} \int_{V} z(x) v_{k}(x) \mathrm{d} x=0 .
$$

Therefore, the identity $\mu=0$ in $V$ holds. Since $\Omega \backslash Z_{\bar{\varphi}}$ is the numerable union of such open sets $V$, we conclude that $\mu$ has its support contained in $Z_{\bar{\varphi}}$. Thus, $\mu \in \mathcal{M}\left(Z_{\bar{\varphi}}\right)$.

We will prove now $\mu^{-}\left(Z_{\bar{\varphi}}^{+}\right)=0$. Let us define the open set $N_{\bar{\varphi}}^{+}:=\cup_{x \in Z_{\bar{\varphi}}^{+}} B_{\rho_{x}}(x) \subset$ $\Omega$, where $\rho_{x}$ is as in the definition of $Z_{\bar{\varphi}}^{+}$. Take $z \in C_{0}\left(Z_{\bar{\varphi}}^{+}\right)$with $z \geq 0$ and extend it continuously by zero to $Z_{\bar{\varphi}}$, which is possible since $Z_{\bar{\varphi}}^{+}$is relatively open in $Z_{\bar{\varphi}}$ by Lemma 2.10. We can further extend $z$ by zero to $\bar{\Omega} \backslash N_{\bar{\varphi}}^{+}$, which makes $z$ continuous on the closed set $\left(\bar{\Omega} \backslash N_{\bar{\varphi}}^{+}\right) \cup Z_{\bar{\varphi}}$. Now, we apply Tietze's extension theorem (see $[21$, Theorem 20.4]), in order to extend $z$ to a function in $C(\bar{\Omega})$. Since $z$ is zero on $\bar{\Omega} \backslash \Omega$ by construction, we have $z \in C_{0}(\Omega)$. Moreover, redefining $z$ as $z^{+}$, we can assume that $z$ is nonnegative and $z=0$ on $\Omega \backslash N_{\bar{\varphi}}^{+}$.

According to (2.10) and the definition of $C_{\bar{u}}^{\tau_{k}}$ we have that $v_{k} \geq 0$ in $N_{\bar{\varphi}}^{+}$. Then, we get

$$
\begin{aligned}
\int_{Z_{\bar{\varphi}}^{+}} z(x) \mathrm{d} \mu(x) & =\int_{Z_{\bar{\varphi}}} z(x) \mathrm{d} \mu(x)=\int_{\Omega} z(x) \mathrm{d} \mu(x) \\
& =\lim _{k \rightarrow \infty} \int_{\Omega} z(x) v_{k}(x) \mathrm{d} x=\lim _{k \rightarrow \infty} \int_{N_{\bar{\varphi}}^{+}} z(x) v_{k}(x) \mathrm{d} x \geq 0 .
\end{aligned}
$$

This shows that

$$
\int_{Z_{\bar{\varphi}}^{+}} z(x) \mathrm{d} \mu(x) \geq 0 \quad \forall z \in C_{0}\left(Z_{\bar{\varphi}}^{+}\right), z \geq 0 .
$$

Hence, we conclude that $\mu$ is a nonnegative measure in $Z_{\bar{\varphi}}^{+}$, and then $\mu^{-}\left(Z_{\bar{\varphi}}^{+}\right)=0$. Analogously we get that $\mu^{+}\left(Z_{\bar{\varphi}}^{-}\right)=0$, which proves that $\mu \in \mathcal{T}_{\bar{u}}$. Thus, we have proved $\mathcal{S}_{\bar{u}} \subset \mathcal{T}_{\bar{u}}$.

In order to prove the inclusion $\mathcal{T}_{\bar{u}} \subset \hat{\mathcal{S}}_{\bar{u}}$, we work with Dirac measures. Here, we exploit the fact that Dirac measures can be obtained as weak-star limits of suitably scaled characteristic functions.

TheOREM 2.12. Suppose that (2.11) holds. Then, the following statements are satisfied:

(1) Let the measure $\mu$ be given by

$$
\begin{gathered}
\mu=\sum_{j=1}^{m} \lambda_{j} \delta_{x_{j}} \quad \text { with pairwise distinct }\left\{x_{j}\right\}_{j=1}^{m} \subset Z_{\bar{\varphi}} \\
\text { and } \lambda_{j} \neq 0 \text { for } 1 \leq j \leq m .
\end{gathered}
$$

Then $\mu$ is in $\mathcal{T}_{\bar{u}}$ if and only if the coefficients $\lambda_{j}$ satisfy for all $j=1, \ldots, m$

$$
\begin{cases}\lambda_{j}>0 & \text { if } x_{j} \in Z_{\bar{\varphi}}^{+}, \\ \lambda_{j}<0 & \text { if } x_{j} \in Z_{\bar{\varphi}}^{-} .\end{cases}
$$


Moreover, the element $\mu$ satisfying (2.33), (2.34) belongs to $\hat{\mathcal{S}}_{\bar{u}}$.

(2) For all $\mu \in \mathcal{T}_{\bar{u}}$ there exists a sequence $\left\{\mu_{k}\right\}_{k=1}^{\infty} \subset \hat{\mathcal{S}}_{\bar{u}}$ satisfying

$$
\mu_{k} \stackrel{*}{\rightarrow} \mu \text { and } \lim _{k \rightarrow \infty}\left\|\mu_{k}\right\|_{\mathcal{M}\left(Z_{\bar{\varphi}}\right)}=\|\mu\|_{\mathcal{M}\left(Z_{\bar{\varphi}}\right)} \text {. }
$$

(3) The set $\hat{\mathcal{S}}_{\bar{u}}$ is sequentially weak-star closed in the following sense: For a sequence $\left\{\mu_{k}\right\}_{k=1}^{\infty} \subset \hat{\mathcal{S}}_{\bar{u}}$ satisfying $\mu_{k} \stackrel{*}{\rightarrow} \mu$ and $\lim _{k \rightarrow \infty}\left\|\mu_{k}\right\|_{\mathcal{M}\left(Z_{\bar{\varphi}}\right)}=\|\mu\|_{\mathcal{M}\left(Z_{\bar{\varphi}}\right)}$ it follows that $\mu \in \hat{\mathcal{S}}_{\bar{u}}$.

(4) It holds that $\mathcal{T}_{\bar{u}} \subset \hat{\mathcal{S}}_{\bar{u}}$.

Proof. (1) Let $\mu$ as in (2.33) be given. We set

$$
I_{+}:=\left\{j: \lambda_{j}>0\right\} \text { and } I_{-}:=\left\{j: \lambda_{j}<0\right\} .
$$

Then, we have

$$
\mu^{+}=\sum_{j \in I_{+}} \lambda_{j} \delta_{x_{j}} \text { and } \mu^{-}=-\sum_{j \in I_{-}} \lambda_{j} \delta_{x_{j}}
$$

We obviously have

$$
\begin{aligned}
& \mu^{-}\left(Z_{\bar{\varphi}}^{+}\right)=0 \quad \Longleftrightarrow \quad x_{j} \notin Z_{\bar{\varphi}}^{+} \quad \forall j \in I_{-}, \\
& \mu^{+}\left(Z_{\bar{\varphi}}^{-}\right)=0 \quad \Longleftrightarrow \quad x_{j} \notin Z_{\bar{\varphi}}^{-} \quad \forall j \in I_{+},
\end{aligned}
$$

which proves that $\mu \in \mathcal{T}_{\bar{u}}$ if and only if (2.34) holds. Let us prove that $\mu$ also belongs to $\hat{\mathcal{S}}_{\bar{u}}$. Let $k_{0}$ be an integer such that

$$
\frac{1}{k_{0}} \leq \min \left\{\left|x_{i}-x_{j}\right|: 1 \leq i, j \leq m, i \neq j\right\}
$$

Now, for every integer $k>2 k_{0}$ we define for $1 \leq j \leq m$

$$
\rho_{j}:=\left\{\begin{array}{cl}
\min \left\{\frac{1}{k}, \rho_{x_{j}}\right\} & \text { if } x_{j} \in Z_{\bar{\varphi}}^{+} \cup Z_{\bar{\varphi}}^{-} \\
\frac{1}{k} & \text { otherwise }
\end{array}\right.
$$

where $\rho_{x_{j}}$ is as in the definition of the sets $Z_{\bar{\varphi}}^{+}$and $Z_{\bar{\varphi}}^{-}$, respectively. We set $r_{k}:=$ $\min _{1 \leq j \leq m} \rho_{j}$ and

$$
v_{k}:=\sum_{x_{j} \in Z_{\bar{\varphi}}^{+} \cup Z_{\bar{\varphi}}^{-}} \frac{\lambda_{j}}{\left|B_{r_{k}}\left(x_{j}\right)\right|} \chi_{B_{r_{k}}\left(x_{j}\right)}+\sum_{x_{j} \notin Z_{\bar{\varphi}}^{+} \cup Z_{\bar{\varphi}}^{-}} \frac{\lambda_{j}}{\left|A_{k}\left(x_{j}\right)\right|} \chi_{A_{k}\left(x_{j}\right)},
$$

where

$$
A_{k}\left(x_{j}\right)= \begin{cases}\left\{x \in B_{r_{k}}\left(x_{j}\right): \bar{\varphi}(x)>0\right\} & \text { if } \lambda_{j}>0, \\ \left\{x \in B_{r_{k}}\left(x_{j}\right): \bar{\varphi}(x)<0\right\} & \text { if } \lambda_{j}<0 .\end{cases}
$$

Note that each $A_{k}\left(x_{j}\right)$ has positive measure since $x_{j} \notin Z_{\bar{\varphi}}^{+} \cup Z_{\bar{\varphi}}^{-}$. If we take

$$
\tau_{k}:=\max \left\{|\bar{\varphi}(x)|: x \in \bigcup_{j=1}^{m} \bar{B}_{r_{k}}\left(x_{j}\right)\right\}
$$


then we have that $\tau_{k} \searrow 0$ and $v_{k} \in C_{\bar{u}}^{\tau_{k}}$. Moreover it is immediate that $v_{k} \stackrel{*}{\rightarrow} \mu$ and

$$
\left\|v_{k}\right\|_{L^{1}(\Omega)}=\sum_{j=1}^{m}\left|\lambda_{j}\right|=\|\mu\|_{\mathcal{M}\left(Z_{\bar{\varphi}}\right)}
$$

which proves $\mu \in \hat{\mathcal{S}}_{\bar{u}}$.

(2) Let $\mu \in \mathcal{T}_{\bar{u}}$ be given. For a given integer $k \geq 1$ we define $\mu_{k} \in \hat{\mathcal{S}}_{\bar{u}}$ as follows. For every point $x \in Z_{\bar{\varphi}}^{+}$we take $\rho_{x} \leq 1 / k$ such that $\bar{\varphi}(\xi) \geq 0$ for all $\xi \in B_{\rho_{x}}(x)$. Analogously we select $\rho_{x}$ for every $x \in Z_{\bar{\varphi}}^{-}$. If $x \in \bar{Z}_{\bar{\varphi}} \backslash\left(Z_{\bar{\varphi}}^{+} \cup Z_{\bar{\varphi}}^{-}\right)$, we take $\rho_{x}=1 / k$. Hence we have that $\bar{Z}_{\bar{\varphi}} \subset \cup_{x \in \bar{Z}_{\bar{\varphi}}} B_{\rho_{x}}(x)$. Since $\bar{Z}_{\bar{\varphi}}$ is a compact subset of $\mathbb{R}^{n}$, we can take a finite subcover $\bar{Z}_{\bar{\varphi}} \subset \cup_{j=1}^{m_{k}} B_{\rho_{j}}\left(x_{j}\right)$, with $\rho_{j}:=\rho_{x_{j}}$. Now, we take a partition of unity subordinated to this cover: $\left\{\phi_{j}\right\}_{j=1}^{m_{k}} \subset C(\bar{\Omega})$ such that

$$
0 \leq \phi_{j}(x) \leq 1 \forall x \in \bar{\Omega}, \quad \sum_{j=1}^{m_{k}} \phi_{j}(x)=1 \forall x \in \bar{Z}_{\bar{\varphi}}, \text { and } \operatorname{supp}\left(\phi_{j}\right) \subset B_{\rho_{j}}\left(x_{j}\right) ;
$$

see [21, Theorem 2.13]. Let us set

$$
\lambda_{j}:= \begin{cases}\int_{\Omega} \phi_{j}(x) \mathrm{d} \mu(x) & \text { if } x_{j} \in Z_{\bar{\varphi}} \\ 0 & \text { if } x_{j} \in \bar{Z}_{\bar{\varphi}} \backslash Z_{\bar{\varphi}}\end{cases}
$$

and

$$
\mu_{k}:=\sum_{j=1}^{m_{k}} \lambda_{j} \delta_{x_{j}} \in \mathcal{M}\left(Z_{\bar{\varphi}}\right)
$$

Let us prove that $\mu_{k} \stackrel{*}{\rightarrow} \mu$. Given $z \in C_{0}\left(Z_{\bar{\varphi}}\right)$ and $\varepsilon>0$ there exists an integer $k_{\varepsilon} \geq 1$ such that

$$
\left|z(x)-z\left(x^{\prime}\right)\right|<\frac{\varepsilon}{|\mu|\left(Z_{\bar{\varphi})}\right.} \forall x, x^{\prime} \in Z_{\bar{\varphi}} \text { such that }\left|x-x^{\prime}\right|<\frac{1}{k_{\varepsilon}} .
$$

Since $z\left(x_{j}\right)=0$ for all $x_{j} \in \bar{Z}_{\bar{\varphi}} \backslash Z_{\bar{\varphi}}$, we have

$$
\int_{Z_{\bar{\varphi}}} z \mathrm{~d} \mu_{k}=\sum_{j=1}^{m_{k}}\left(\int_{\Omega} \phi_{j}(x) \mathrm{d} \mu(x)\right) z\left(x_{j}\right)
$$

Then we infer for $k \geq k_{\varepsilon}$ that

$$
\left|\int_{Z_{\bar{\varphi}}} z(x) \mathrm{d} \mu_{k}(x)-\int_{Z_{\bar{\varphi}}} z(x) \mathrm{d} \mu(x)\right| \leq \sum_{j=1}^{m_{k}} \int_{Z_{\bar{\varphi}}} \phi_{j}(x)\left|z\left(x_{j}\right)-z(x)\right| \mathrm{d}|\mu|(x) \leq \varepsilon,
$$

which proves the desired convergence. To prove that $\mu_{k} \in \hat{\mathcal{S}}_{\bar{u}}$ it is enough to use statement (1) of the theorem and take into account that if $x_{j} \in Z_{\bar{\varphi}}^{+}$, then $\mu^{-}=0$ in $B_{\rho_{j}}\left(x_{j}\right)$ and

$$
\int_{Z_{\bar{\varphi}}} \phi_{j}(x) \mathrm{d} \mu(x)=\int_{Z_{\bar{\varphi}} \cap B_{\rho_{j}}\left(x_{j}\right)} \phi_{j}(x) \mathrm{d} \mu^{+}(x) \geq 0 .
$$

Similarly we proceed with the points $x_{j} \in Z_{\bar{\varphi}}^{-}$. Thus we conclude that $\mu_{k} \in \hat{\mathcal{S}}_{\bar{u}}$ for every $k$. It remains to prove the convergence of norms $\left\|\mu_{k}\right\|_{\mathcal{M}\left(Z_{\bar{\varphi}}\right)} \rightarrow\|\mu\|_{\mathcal{M}\left(Z_{\bar{\varphi}}\right)}$. First we notice that

$$
\left\|\mu_{k}\right\|_{\mathcal{M}\left(Z_{\bar{\varphi}}\right)} \leq \sum_{j=1}^{m_{k}}\left|\int_{Z_{\bar{\varphi}}} \phi_{j}(x) \mathrm{d} \mu(x)\right| \leq \int_{Z_{\bar{\varphi}}} \mathrm{d}|\mu|(x)=\|\mu\|_{\mathcal{M}\left(Z_{\bar{\varphi}}\right)} .
$$


From here we infer that

$$
\|\mu\|_{\mathcal{M}\left(Z_{\bar{\varphi}}\right)} \leq \liminf _{k \rightarrow \infty}\left\|\mu_{k}\right\|_{\mathcal{M}\left(Z_{\bar{\varphi}}\right)} \leq \limsup _{k \rightarrow \infty}\left\|\mu_{k}\right\|_{\mathcal{M}\left(Z_{\bar{\varphi}}\right)} \leq\|\mu\|_{\mathcal{M}\left(Z_{\bar{\varphi}}\right)} .
$$

Thus, $\left\|\mu_{k}\right\|_{\mathcal{M}\left(Z_{\bar{\varphi}}\right)} \rightarrow\|\mu\|_{\mathcal{M}\left(Z_{\bar{\varphi}}\right)}$ and this proves (2.35).

(3) Let a sequence $\left\{\mu_{k}\right\}_{k=1}^{\infty} \subset \hat{\mathcal{S}}_{\bar{u}}$ with $\mu_{k} \stackrel{*}{\rightarrow} \mu$ and $\lim _{k \rightarrow \infty}\left\|\mu_{k}\right\|_{\mathcal{M}\left(Z_{\bar{\varphi}}\right)}=$ $\|\mu\|_{\mathcal{M}\left(Z_{\bar{\varphi}}\right)}$ be given. By definition of $\hat{\mathcal{S}}_{\bar{u}}$ in $(2.32)$ there are sequences $\left\{v_{k, i}\right\}_{i=1}^{\infty}$, $\left\{\tau_{k, i}\right\}_{i=1}^{\infty}$ with $v_{k, i} \in C_{\bar{u}}^{\tau_{k, i}}$ and $\tau_{k, i} \searrow 0$ as $i \rightarrow \infty$ and such that

$$
v_{k, i} \stackrel{*}{\rightarrow} \mu_{k} \text { and }\left\|v_{k, i}\right\|_{L^{1}(\Omega)} \rightarrow\left\|\mu_{k}\right\|_{\mathcal{M}\left(Z_{\bar{\varphi}}\right)} \quad \text { as } i \rightarrow \infty .
$$

Hence, all these sequences (at least if we skip some of the first elements) belong to a large ball in $\mathcal{M}\left(Z_{\bar{\varphi}}\right)$. Indeed, since $\lim _{k \rightarrow \infty}\left\|\mu_{k}\right\|_{\mathcal{M}\left(Z_{\bar{\varphi}}\right)}=\|\mu\|_{\mathcal{M}\left(Z_{\bar{\varphi}}\right)}$, we can take a real number $r>0$ such that $\left\|\mu_{k}\right\|_{\mathcal{M}}\left(Z_{\bar{\varphi}}\right) \leq r / 2$ for all $k \geq 1$. Now, from the convergence $\left\|v_{k, i}\right\|_{L^{1}(\Omega)} \rightarrow \mu_{k}$ we infer the existence of $i_{k} \geq 1$ such that $\left\|v_{k, i}\right\|_{L^{1}(\Omega)}<r$ for all $i \geq i_{k}$. Thus, we conclude that $\left\{v_{k, i}\right\}_{i \geq i_{k}} \subset B_{r}(0)$ and $\left\{\mu_{k}\right\}_{k=1}^{\infty} \subset B_{r}(0)$, where $B_{r}(0)$ denotes the ball in $\mathcal{M}\left(Z_{\bar{\varphi}}\right)$ of radius $r$ centered at 0 . On this ball, the weak-star topology is metrizable (see, for instance, [3, Theorem 3.28]) and, thus, we can pick a diagonal sequence $\left\{v_{k, i_{k}}\right\}_{k=1}^{\infty}$ which converges to $\mu$ weakly star in $\mathcal{M}\left(Z_{\bar{\varphi}}\right)$ and such that $\tau_{k, i_{k}} \searrow 0$ and $\left\|v_{k, i_{k}}\right\|_{L^{1}(\Omega)} \rightarrow\|\mu\|_{\mathcal{M}\left(Z_{\bar{\varphi}}\right)}$. This implies $\mu \in \hat{\mathcal{S}}_{\bar{u}}$, which is the claim.

(4) The inclusion $\mathcal{T}_{\bar{u}} \subset \hat{\mathcal{S}}_{\bar{u}}$ is an immediate consequence of statements (2) and (3).

From Lemma 2.11 and Theorem 2.12 we infer the following

Corollary 2.13. Suppose that (2.11) holds. Then it holds that $\mathcal{T}_{\bar{u}}=\mathcal{S}_{\bar{u}}=\hat{\mathcal{S}}_{\bar{u}}$. Moreover, this set is sequentially weak-star closed in $\mathcal{M}\left(Z_{\bar{\varphi}}\right)$.

Proof. From the definitions (2.31) and (2.32), we get $\hat{\mathcal{S}}_{\bar{u}} \subset \mathcal{S}_{\bar{u}}$. In Lemma 2.11, we have shown that $\mathcal{S}_{\bar{u}} \subset \mathcal{T}_{\bar{u}}$ and Theorem 2.12(4) yields $\mathcal{T}_{\bar{u}} \subset \hat{\mathcal{S}}_{\bar{u}}$. This shows that $\mathcal{T}_{\bar{u}}=\mathcal{S}_{\bar{u}}=\hat{\mathcal{S}}_{\bar{u}}$.

Now, let the sequence $\left\{\mu_{k}\right\}_{k=1}^{\infty} \subset \hat{\mathcal{S}}_{\bar{u}}$ converge towards $\mu$ w.r.t. the weak-star topology of $\mathcal{M}\left(Z_{\bar{\varphi}}\right)$. This implies the boundedness of $\left\{\left\|\mu_{k}\right\|_{\mathcal{M}\left(Z_{\bar{\varphi}}\right)}\right\}_{k=1}^{\infty}$. Now, we can argue as in the proof of Theorem 2.12(3) to obtain a diagonal sequence $\left\{v_{k, i_{k}}\right\}_{k=1}^{\infty}$ with $v_{k, i_{k}} \in C_{\bar{u}}^{\tau_{k}, i_{k}}$ and $v_{k, i_{k}} \stackrel{*}{\rightarrow} \mu$.

Note that, different from the proof of Theorem 2.12(3), we do not obtain the convergence of norms $\left\|v_{k, i_{k}}\right\|_{L^{1}(\Omega)} \rightarrow\|\mu\|_{\mathcal{M}\left(Z_{\bar{\varphi}}\right)}$ as $k \rightarrow \infty$ since we do not have $\left\|\mu_{k}\right\|_{\mathcal{M}\left(Z_{\bar{\varphi}}\right)} \rightarrow\|\mu\|_{\mathcal{M}\left(Z_{\bar{\varphi}}\right)}$.

Nevertheless, the above arguments show that $\mu \in \mathcal{S}_{\bar{u}}$ and, together with $\mathcal{S}_{\bar{u}}=\hat{\mathcal{S}}_{\bar{u}}$, this shows the claim.

The equivalence between the conditions (2.25) and (2.30) is an immediate consequence of the following theorem.

THEOREM 2.14. Suppose that assumptions (A1)-(A3) and (2.11) hold. Then, the conditions

$$
\begin{aligned}
\exists \tau>0, \exists \kappa^{\prime}>0: & J^{\prime \prime}(\bar{u}) v^{2} \geq-\kappa^{\prime}\|v\|_{L^{1}(\Omega)}^{2} & \forall v \in C_{\bar{u}}^{\tau}, \\
\exists \kappa^{\prime \prime}>0: & J^{\prime \prime}(\bar{u}) \mu^{2} \geq-\kappa^{\prime \prime}\|\mu\|_{\mathcal{M}\left(Z_{\bar{\varphi}}\right)}^{2} & \forall \mu \in \mathcal{T}_{\bar{u}}
\end{aligned}
$$

are equivalent. Moreover, if (2.37) holds for a constant $\kappa^{\prime \prime}$, then (2.36) is valid for any constant $\kappa^{\prime}>\kappa^{\prime \prime}$. Reciprocally, if (2.36) holds for $\kappa^{\prime}$, then (2.37) is fulfilled for the same constant $\kappa^{\prime}$. 
Proof. We first prove that (2.37) implies (2.36). We will argue by contradiction. To this end, suppose that (2.37) is fulfilled for a constant $\kappa^{\prime \prime}$, but (2.36) does not hold for some $\kappa^{\prime}>\kappa^{\prime \prime}$. Since (2.36) does not hold for the constant $\kappa^{\prime}$ and any $\tau>0$, then there are sequences $\left\{\tau_{k}\right\}_{k=1}^{\infty} \subset \mathbb{R}$ and $\left\{v_{k}\right\}_{k=1}^{\infty} \subset L^{1}(\Omega)$ such that

$$
\left\|v_{k}\right\|_{L^{1}(\Omega)}=1, \quad \tau_{k} \searrow 0, \quad v_{k} \in C_{\bar{u}}^{\tau_{k}}, \quad J^{\prime \prime}(\bar{u}) v_{k}^{2}<-\kappa^{\prime} .
$$

The boundedness of $\left\{v_{k}\right\}_{k=1}^{\infty}$ in $L^{1}(\Omega) \subset \mathcal{M}(\Omega)$ implies the existence of an element $\mu \in \mathcal{M}(\Omega)$ and a subsequence, denoted the same, such that $v_{k} \stackrel{*}{\rightarrow} \mu$ in $\mathcal{M}(\Omega)$. Hence, it holds that $\mu \in \mathcal{S}_{\bar{u}}$. From Lemma 2.11 we know that $\mu \in \mathcal{S}_{\bar{u}} \subset \mathcal{T}_{\bar{u}}$. Hence, (2.37) is satisfied by $\mu$. Because of the compactness of the embedding $W_{0}^{1, p}(\Omega) \hookrightarrow L^{2}(\Omega)$ for $p>\frac{2 n}{n+2}$, the convergence $v_{k} \stackrel{*}{\rightarrow} \mu$ in $\mathcal{M}(\Omega)$ implies $z_{v_{k}} \rightarrow z_{\mu}$ in $L^{2}(\Omega)$ (see also [16, Lemma 2.15]), and $J^{\prime \prime}(\bar{u}) v_{k}^{2} \rightarrow J^{\prime \prime}(\bar{u}) \mu^{2}$. Hence, we obtain $J^{\prime \prime}(\bar{u}) \mu^{2} \leq-\kappa^{\prime}$ and $\mu \neq 0$. But by (2.37) it holds that $J^{\prime \prime}(\bar{u}) \mu^{2} \geq-\kappa^{\prime \prime}\|\mu\|_{\mathcal{M}\left(Z_{\bar{\varphi}}\right)}^{2}$. This leads to

$$
\|\mu\|_{\mathcal{M}\left(Z_{\bar{\varphi}}\right)} \geq \frac{\kappa^{\prime}}{\kappa^{\prime \prime}}>1
$$

which is a contradiction to $\|\mu\|_{\mathcal{M}\left(Z_{\bar{\varphi}}\right)} \leq \liminf _{k \rightarrow \infty}\left\|v_{k}\right\|_{L^{1}(\Omega)}=1$.

Let us prove the converse implication. Given $\mu \in \mathcal{T}_{\bar{u}}$ we get from Theorem 2.12(4) that $\mu$ belongs to $\hat{\mathcal{S}}_{\bar{u}}$. Hence, there exist sequences $\left\{\tau_{k}\right\}_{k=1}^{\infty} \in \mathbb{R}$ and $\left\{v_{k}\right\}_{k=1}^{\infty} \in L^{1}(\Omega)$ such that

$$
\tau_{k} \searrow 0, \quad v_{k} \in C_{\bar{u}}^{\tau_{k}}, v_{k} \stackrel{*}{\rightarrow} \mu, \quad \text { and }\left\|v_{k}\right\|_{L^{1}(\Omega)} \rightarrow\|\mu\|_{\mathcal{M}\left(Z_{\bar{\varphi}}\right)} .
$$

Again, we obtain $J^{\prime \prime}(\bar{u}) v_{k}^{2} \rightarrow J^{\prime \prime}(\bar{u}) \mu^{2}$. From (2.36) we conclude that there is $\kappa^{\prime}$ such that for all $k$ sufficiently large the inequality

$$
J^{\prime \prime}(\bar{u}) v_{k}^{2} \geq-\kappa^{\prime}\left\|v_{k}\right\|_{L^{1}(\Omega)}^{2}
$$

is satisfied. Passing to the limit $k \rightarrow \infty$ shows that (2.37) is satisfied with $\kappa^{\prime \prime}=\kappa^{\prime}$.

Using Theorem 2.8, we obtain the following corollary.

Corollary 2.15. Suppose that assumptions (A1)-(A4) hold. Further, we assume that (2.30) is satisfied, where the constant $\kappa$ is given in Proposition 2.7. Then, for all $\kappa^{\prime \prime} \in\left(\kappa^{\prime}, \kappa\right)$ there exists $\varepsilon>0$ such that

$$
J(\bar{u})+\frac{\kappa-\kappa^{\prime \prime}}{8}\|u-\bar{u}\|_{L^{1}(\Omega)}^{2} \leq J(u) \quad \forall u \in \mathcal{U}_{\mathrm{ad}} \cap B_{\varepsilon}^{1}(\bar{u}) .
$$

Remark 2.16. Let us assume that $\bar{u} \in \mathcal{U}_{\text {ad }}$ satisfies the first-order optimality conditions (2.7)-(2.9) and (2.11), but the structural assumption (2.16) is not satisfied. If condition (2.14) holds, the following statement is satisfied:

$$
\exists \kappa>0 \text { such that } J^{\prime \prime}(\bar{u}) \mu^{2} \geq \kappa\left\|z_{\mu}\right\|_{L^{2}(\Omega)}^{2} \quad \forall \mu \in \mathcal{T}_{\bar{u}} .
$$

The proof follows the steps of the second part of the proof of Theorem 2.14. We have to replace $-\kappa^{\prime}$ by $\kappa$ in (2.39) as well as the norms $\left\|v_{k}\right\|_{L^{1}(\Omega)}$ and $\|\mu\|_{\mathcal{M}\left(Z_{\bar{\varphi}}\right)}$ by $\left\|z_{v_{k}}\right\|_{L^{2}(\Omega)}$ and $\left\|z_{\mu}\right\|_{L^{2}(\Omega)}$, respectively.

Remark 2.17. We compare our results with those obtained in [6] in the case that (2.16) is satisfied. First of all, it is clear that condition (2.14), which is needed in [6], immediately implies our condition (2.25) (with $\kappa^{\prime}=0$ ). Then, due to $\left\|z_{v}\right\|_{L^{2}(\Omega)} \leq$ 
$C_{1}\|v\|_{L^{1}(\Omega)}$, the quadratic growth $(2.26)$ in $L^{1}(\Omega)$ implies the quadratic growth w.r.t. the $L^{2}(\Omega)$ norm of the state, i.e., (2.15) is satisfied; see also [6, Proof of Corollary 2.8].

Hence, in the case that (2.16) is satisfied, our assumptions are weaker than those of [6] and we obtain a stronger result. However, we emphasize that [6] is also applicable in the case that (2.16) does not hold.

Due to the structure of $J^{\prime \prime}(\bar{u})$, it seems more natural to formulate a secondorder condition in terms of $\left\|z_{v}\right\|_{L^{2}(\Omega)}^{2}$ or $\left\|z_{\mu}\right\|_{L^{2}(\Omega)}^{2}$ instead of $\|v\|_{L^{1}(\Omega)}^{2}$ or $\|\mu\|_{\mathcal{M}\left(Z_{\bar{\varphi}}\right)}^{2}$. However, observe that the second-order condition

$$
J^{\prime \prime}(\bar{u}) v^{2} \geq-\delta\left\|z_{v}\right\|_{L^{2}(\Omega)}^{2} \quad \forall v \in C_{\bar{u}}^{\tau}
$$

with $\delta \leq \frac{\kappa^{\prime}}{C_{1}^{2}}$ implies (2.25). Analogously, under the same assumption on $\delta$, the condition

$$
J^{\prime \prime}(\bar{u}) \mu^{2} \geq-\delta\left\|z_{\mu}\right\|_{L^{2}(\Omega)}^{2} \quad \forall \mu \in \mathcal{T}_{\bar{u}}
$$

implies (2.30). According to Theorem 2.8 and Corollary 2.15, the above second-order conditions are sufficient for $L^{2}(\Omega)$ local optimality of $\bar{u}$ if $C_{1}^{2} \delta<\kappa$ holds.

3. Abstract setting. The technique of section 2 can also be transferred to an abstract setting. In fact, it is sufficient to pose some assumptions on the reduced objective $J: L^{\infty}(X) \rightarrow \mathbb{R}$. We will briefly address the necessary modifications in this section. Throughout this section, we assume that $(X, \mathcal{B}, \eta)$ is a finite and complete measure space.

We consider the abstract problem

$$
\begin{aligned}
\text { Minimize } & J(u) \\
\text { w.r.t. } & u \in L^{\infty}(X) \\
\text { such that } & \alpha \leq u \leq \beta .
\end{aligned}
$$

Here, $J: \mathcal{U}_{\text {ad }} \rightarrow \mathbb{R}$ is a given function, where $\mathcal{U}_{\text {ad }} \subset L^{\infty}(X)$ is defined analogously to section 2 using the bounds $\alpha, \beta \in L^{\infty}(X)$.

Let $\bar{u} \in \mathcal{U}_{\text {ad }}$ be fixed. We make the following assumptions on $J$ and $\bar{u}$ :

(H1) The functional $J$ can be extended to an $L^{\infty}(X)$-neighborhood of $\mathcal{U}_{\text {ad }}$. It is twice continuously Fréchet differentiable w.r.t. $L^{\infty}(X)$ in this neighborhood. Moreover, we assume that $\bar{u}$ satisfies the first-order condition $J^{\prime}(\bar{u})(u-\bar{u}) \geq 0$ for all $u \in \mathcal{U}_{\text {ad }}$.

(H2) There is $\delta>0$ such that the second derivative $J^{\prime \prime}(u): L^{\infty}(X)^{2} \rightarrow \mathbb{R}$ can be extended continuously to $L^{q}(X)^{2}$, for some $q \in[1,3 / 2)$, for all $u \in \mathcal{U}_{\text {ad }} \cap$ $B_{\delta}^{1}(\bar{u})$. In particular, there is a constant $C>0$ such that

$$
\left|J^{\prime \prime}(\bar{u})\left(v_{1}, v_{2}\right)\right| \leq C\left\|v_{1}\right\|_{L^{q}(X)}\left\|v_{2}\right\|_{L^{q}(X)}
$$

holds for all $v_{1}, v_{2} \in L^{q}(X)$.

(H3) For each $\varepsilon>0$, there is $\delta>0$ such that

$$
\left|\left[J^{\prime \prime}\left(u_{\theta}\right)-J^{\prime \prime}(\bar{u})\right](u-\bar{u})^{2}\right| \leq \varepsilon\|u-\bar{u}\|_{L^{1}(X)}^{2}
$$

holds for all $u \in \mathcal{U}_{\mathrm{ad}} \cap B_{\delta}^{1}(\bar{u})$ and $u_{\theta} \in \mathcal{U}_{\mathrm{ad}}$ with $\left\|u_{\theta}-\bar{u}\right\|_{L^{1}(X)} \leq\|u-\bar{u}\|_{L^{1}(X)}$.

(H4) There exists a function $\bar{\varphi} \in L^{1}(X)$ such that $J^{\prime}(\bar{u}) v=\int_{X} \bar{\varphi} v \mathrm{~d} \eta$ for all $v \in L^{\infty}(X)$. 
(H5) There exists a constant $K>0$ such that

$$
\eta(\{x \in X:|\bar{\varphi}(x)| \leq \varepsilon\}) \leq K \varepsilon
$$

is satisfied for all $\varepsilon>0$.

We briefly discuss that the above assumptions are satisfied for the optimal control problem which is discussed in section 2. Assumptions (H1)-(H4) are satisfied with $q=$ 1 , which can be seen from (2.27) and (2.28) in the proof of Theorem 2.8. Assumption (H5) translates to (A4).

It is easy to see that Proposition 2.7 can be directly transferred to this abstract situation. In order to use the proof of Theorem 2.8 in this abstract setting, we have to show that (3.1) yields an estimate against $L^{1}(X)$-norms. This follows directly by Hölder's inequality.

Corollary 3.1. Let assumptions (H1)-(H2) be satisfied. Then, there is a constant $C>0$ such that

$$
\left|J^{\prime \prime}(\bar{u})\left(v_{1}, v_{2}\right)\right| \leq C\left\|v_{1}\right\|_{L^{1}(X)}^{1 / q}\left\|v_{2}\right\|_{L^{1}(X)}^{1 / q}\left\|v_{1}\right\|_{L^{\infty}(X)}^{(q-1) / q}\left\|v_{2}\right\|_{L^{\infty}(X)}^{(q-1) / q}
$$

holds for all $v_{1}, v_{2} \in L^{\infty}(X)$.

Next, we give two possibilities to verify (H3).

Lemma 3.2. Let assumptions (H1)-(H2) be satisfied. Then, (H3) follows if one of the following conditions is satisfied:

(1) For each $\varepsilon>0$, there is $\delta>0$ such that

$$
\left|\left[J^{\prime \prime}(u)-J^{\prime \prime}(\bar{u})\right] v^{2}\right| \leq \varepsilon\|v\|_{L^{1}(X)}^{2}
$$

holds for all $u \in \mathcal{U}_{\mathrm{ad}} \cap B_{\delta}^{1}(\bar{u})$ and $v \in L^{\infty}(X)$.

(2) Let $p \in[1, \infty]$ and $r \in[q, 2)$ satisfy $1 / p+2 / r>2$ with $q$ as in (H2). Moreover, assume that there exist positive constants $\delta_{0}$ and $C$ such that

$$
\left|\left[J^{\prime \prime}(u)-J^{\prime \prime}(\bar{u})\right] v^{2}\right| \leq C\|u-\bar{u}\|_{L^{p}(X)}\|v\|_{L^{r}(X)}^{2}
$$

holds for all $u \in \mathcal{U}_{\mathrm{ad}} \cap B_{\delta_{0}}^{1}(\bar{u})$ and $v \in L^{\infty}(X)$.

Proof. The verification of (H3) in case (1) is straightforward.

Let us suppose that (2) holds. From Hölder's inequality we get for $u \in \mathcal{U}_{\mathrm{ad}} \cap B_{\delta_{0}}^{1}(\bar{u})$

$$
\begin{aligned}
\left|\left[J^{\prime \prime}\left(u_{\theta}\right)-J^{\prime \prime}(\bar{u})\right](u-\bar{u})^{2}\right| & \leq C\left\|u_{\theta}-\bar{u}\right\|_{L^{p}(X)}\|u-\bar{u}\|_{L^{r}(X)}^{2} \\
& \leq C\left\|u_{\theta}-\bar{u}\right\|_{L^{1}(X)}^{1 / p}\|u-\bar{u}\|_{L^{1}(X)}^{2 / r} \leq C\|u-\bar{u}\|_{L^{1}(X)}^{1 / p+2 / r} .
\end{aligned}
$$

Since $1 / p+2 / r>2$, the claim follows.

Using these assumptions above, we can prove the next theorem, following along the lines of the proof of Theorem 2.8.

THEOREM 3.3. Suppose that the above assumptions (H1)-(H5) are satisfied. Further, we assume that

$$
\exists \tau>0, \exists \kappa^{\prime}<\kappa: \quad J^{\prime \prime}(\bar{u}) v^{2} \geq-\kappa^{\prime}\|v\|_{L^{1}(X)}^{2} \quad \forall v \in C_{\bar{u}}^{\tau},
$$

where $\kappa=\left(4\|\beta-\alpha\|_{L^{\infty}(X)} K\right)^{-1}$; cf. Proposition 2.7. Then, there exists $\varepsilon>0$ such that

$$
J(\bar{u})+\frac{\kappa-\kappa^{\prime}}{8}\|u-\bar{u}\|_{L^{1}(X)}^{2} \leq J(u) \quad \forall u \in \mathcal{U}_{\mathrm{ad}} \cap B_{\varepsilon}^{1}(\bar{u}) .
$$


Proof. We follow the proof of Theorem 2.8 and only show the necessary modifications. Substituting (2.27) by (H3), we obtain

$$
J(u)-J(\bar{u}) \geq \frac{\kappa+\kappa^{\prime}}{4}\|u-\bar{u}\|_{L^{1}(X)}^{2}+\frac{\tau}{2}\|u-\bar{u}\|_{L^{1}\left(X_{\tau}\right)}+\frac{1}{2} J^{\prime \prime}(\bar{u})(u-\bar{u})^{2}
$$

for all $u \in B_{\varepsilon_{0}}^{1}(\bar{u})$. Here, $X_{\tau}=\{x \in X:|\bar{\varphi}(x)|>\tau\}$. Again we will use the splitting $u=u_{1}+u_{2}$ with $u_{1}-\bar{u} \in C_{\bar{u}}^{\tau}$. Then, by applying (3.5), Corollary 3.1, and Young's inequality, we find

$$
\begin{aligned}
\frac{1}{2} J^{\prime \prime}(\bar{u})(u-\bar{u})^{2} & =\frac{1}{2} J^{\prime \prime}(\bar{u})\left(u_{1}-\bar{u}\right)^{2}+\frac{1}{2} J^{\prime \prime}(\bar{u}) u_{2}^{2}+J^{\prime \prime}(\bar{u})\left(u_{1}-\bar{u}, u_{2}\right) \\
& \geq-\frac{\kappa^{\prime}}{2}\left\|u_{1}-\bar{u}\right\|_{L^{1}(X)}^{2}-C\left\|u_{1}-\bar{u}\right\|_{L^{1}(X)}^{1 / q}\left\|u_{2}\right\|_{L^{1}(X)}^{1 / q}-C\left\|u_{2}\right\|_{L^{1}(X)}^{2 / q} \\
& \geq-\frac{\kappa+3 \kappa^{\prime}}{8}\left\|u_{1}-\bar{u}\right\|_{L^{1}(X)}^{2}-C\left\|u_{2}\right\|_{L^{1}(X)}^{2 /(2 q-1)}-C\left\|u_{2}\right\|_{L^{1}(X)}^{2 / q} .
\end{aligned}
$$

Owing to the construction of $u_{1}$ and $u_{2}$, we have

$$
\frac{1}{2} J^{\prime \prime}(\bar{u})(u-\bar{u})^{2} \geq-\frac{\kappa+3 \kappa^{\prime}}{8}\|u-\bar{u}\|_{L^{1}(X)}^{2}-C\|u-\bar{u}\|_{L^{1}\left(X_{\tau}\right)}^{\bar{q}}
$$

with $\bar{q}=\min (2 /(2 q-1), 2 / q)=2 /(2 q-1)>1$, since $q<3 / 2$. This implies

$$
J(u)-J(\bar{u}) \geq \frac{\kappa-\kappa^{\prime}}{8}\|u-\bar{u}\|_{L^{1}(X)}^{2}+\left(\frac{\tau}{2}-C\|u-\bar{u}\|_{L^{1}\left(X_{\tau}\right)}^{\bar{q}-1}\right)\|u-\bar{u}\|_{L^{1}\left(X_{\tau}\right)},
$$

and the claim follows immediately.

In order to provide a second-order condition based on measures as in Corollary 2.15 , we need to extend the first and second derivatives of $J$ to measures. Therefore, we additionally assume the following:

(H6) There exists a topology $\tau$ on $X$ such that $(X, \tau)$ is a locally compact, metrizable, and $\sigma$-compact Hausdorff space.

(H7) The $\sigma$-algebra $\mathcal{B}$ contains all Borel sets of $(X, \tau)$ and $\operatorname{supp}(\eta)=X$.

(H8) $\bar{\varphi} \in C_{0}(X)$. Hence, $J^{\prime}(\bar{u})$ can be extended continuously to $\mathcal{M}(X)$.

(H9) The bilinear form $J^{\prime \prime}(\bar{u})$ on $L^{\infty}(X)^{2}$ can be extended to a weak-star sequentially continuous bilinear form $J^{\prime \prime}(\bar{u})$ on $\mathcal{M}(X)^{2}$.

We briefly give some comments. First, we recall that $(X, \tau)$ is $\sigma$-compact if $X$ can be written as a countable union of compact sets. This assumption implies that $C_{0}(X)$ is separable; see Lemma 3.4 below. Following [21, Definition 3.16], $C_{0}(X)$ denotes the Banach space of continuous functions $f: X \longrightarrow \mathbb{R}$ such that for every $\varepsilon>0$ there exists a compact set $F \subset X$ such that $|f(x)|<\varepsilon$ for all $x \in X \backslash F$.

The second assumption (H7) is a compatibility condition between the measure space structure and the metric structure on $X$. The last two assertions (H8) and (H9) allow us to extend the derivatives of the objective $J$ to measures. We also recall that $\mathcal{M}(X)$ can be identified with the dual space of $C_{0}(X)$ under the natural duality mapping

$$
\langle f, \mu\rangle=\int_{X} f \mathrm{~d} \mu \quad \forall f \in C_{0}(X), \mu \in \mathcal{M}(X) ;
$$

see [21, Theorem 6.19].

Lemma 3.4. Under assumption (H6), the space $C_{0}(X)$ is separable. 
Proof. From [12, Theorem 5.3] we find that the one-point compactification $\tilde{X}$ of $X$ is compact metrizable. Hence, [12, Theorem 4.19] implies that $C(\tilde{X})$ is separable. Since $C_{0}(X)$ is a closed subspace of $C(\tilde{X})$, the assertion follows.

Now, one can verify that the results Lemmas 2.10 and 2.11, Theorem 2.12, and Corollary 2.13 can be transferred to the abstract situations; in the proofs one has to replace $\bar{\Omega}$ by the one-point compactification $\tilde{X}$ of $X$. Moreover, the Euclidean metric on $\bar{\Omega}$ is replaced by a metric on $\tilde{X}$, which is metrizable due to [12, Theorem 5.3]. Note that the second part of (H7) is needed for the proof of Theorem 2.12(1) in order to guarantee that all occurring balls have positive measure w.r.t. $\eta$. Similarly, the proof of Theorem 2.14 carries over by using (H9). Thus, we obtain the following analogue to Corollary 2.15 .

TheOrem 3.5. Suppose that the above assumptions are satisfied. Further, we assume that

$$
\exists \kappa^{\prime}<\kappa: \quad J^{\prime \prime}(\bar{u}) \mu^{2} \geq-\kappa^{\prime}\|\mu\|_{\mathcal{M}\left(Z_{\bar{\varphi}}\right)}^{2} \forall \mu \in \mathcal{T}_{\bar{u}}
$$

is satisfied, where the constant $\kappa$ is given in Proposition 2.7. Then, there exists $\varepsilon>0$ such that

$$
J(\bar{u})+\frac{\kappa-\kappa^{\prime}}{16}\|u-\bar{u}\|_{L^{1}(X)}^{2} \leq J(u) \quad \forall u \in \mathcal{U}_{\mathrm{ad}} \cap B_{\varepsilon}^{1}(\bar{u}) .
$$

We finish this section by showing some examples that fall into the previous abstract framework.

Example 1. Under the notation of section 1 we define the boundary elliptic control problem

$$
\begin{cases}\text { Minimize } & J(u):=\int_{\Omega} L\left(x, y_{u}(x)\right) \mathrm{d} x \\ \text { such that } & \alpha(x) \leq u(x) \leq \beta(x) \quad \text { for a.a. } x \in \Gamma,\end{cases}
$$

where $y_{u}$ is the weak solution of the elliptic boundary value problem

$$
\left\{\begin{aligned}
A y+a(\cdot, y)=0 & \text { in } \Omega, \\
\partial_{n_{A}} y=u & \text { on } \Gamma .
\end{aligned}\right.
$$

Above, $\partial_{n_{A}} y$ denotes the normal derivative associated to the operator $A$, formally

$$
\partial_{n_{A}} y=\sum_{i, j=1}^{n} a_{i j} \partial_{x_{i}} y n_{j}
$$

with $\mathbf{n}(x)=\left\{n_{j}(x)\right\}_{j=1}^{n}$ denoting the exterior unit vector to $\Gamma$ at the point $x$. Besides assumptions (A1)-(A3) we suppose that there exists a measurable set $F_{a} \subset \Omega$ with $\left|F_{a}\right|>0$ such that $\partial_{y} a(x, t)>0$ for all $(x, t) \in F_{a} \times \mathbb{R}$. This assumption is required to deduce the existence and uniqueness of a solution of the state equation and the linearized equations.

Taking $X=\Gamma$ and $\eta$ as the usual measure on the Lipschitz manifold $\Gamma$, this control problem fits in the above abstract framework. Let us point out that to check hypothesis (H9) it is enough to use the regularity results of the solution of a Neumann problem with a measure as datum on the boundary; see, e.g., [4]. 
Example 2. Now we consider the following parabolic control problem:

$$
\begin{cases}\text { Minimize } & J(u):=\int_{Q} L\left(x, t, y_{u}(x, t)\right) \mathrm{d} x \mathrm{~d} t \\ \text { such that } \quad \alpha_{j}(t) \leq u_{j}(t) \leq \beta_{j}(t) \quad \text { for a.a. } t \in(0, T), \quad 1 \leq j \leq m\end{cases}
$$

where $y_{u}$ is the weak solution of the parabolic boundary value problem

$$
\left\{\begin{aligned}
\partial_{t} y+A y+a(\cdot, \cdot, y) & =\sum_{j=1}^{m} u_{j}(t) g_{j}(x) \quad \text { in } Q=\Omega \times(0, T), \\
y(0) & =y_{0} \quad \text { in } \Omega \\
y & =0 \quad \text { on } \Sigma=\Gamma \times(0, T),
\end{aligned}\right.
$$

where $y_{0} \in C_{0}(\Omega),\left\{g_{j}\right\}_{j=1}^{m} \subset L^{p}(\Omega)$ with $p>n$ are given, $\alpha_{j}, \beta_{j} \in L^{\infty}(0, T)$ for $1 \leq j \leq m$, and $u=\left\{u_{j}\right\}_{j=1}^{m}$ denote the controls. We assume that $\operatorname{supp}\left(g_{j}\right)=\omega_{j} \subset \Omega$, the sets $\left\{\omega_{j}\right\}_{j=1}^{m}$ being pairwise disjoint. Then, the state equation has a unique solution $y_{u} \in L^{2}\left(0, T ; H_{0}^{1}(\Omega)\right) \cap C(\bar{Q})$. This problem falls in the abstract framework if we take $X=\{1, \ldots, m\} \times(0, T)$ and if $\eta$ consists of $m$ copies of the one-dimensional Lebesgue measure. Once again, we have to pay attention to hypothesis (H9). We recall that if the right-hand side of a linear parabolic equation is a measure $\mu \in \mathcal{M}(Q)$, then the solution $z_{\mu}$ does not belong to $L^{2}(Q)$. Therefore, the case of distributed controls $u \in L^{2}(Q)$ does not fall into the abstract framework because (H9) is not fulfilled. However, in the above problem we have considered controls depending only on time. In this case, if we take $\mu \in \mathcal{M}(X)=\mathcal{M}\left((0, T)^{m}\right)$ and the functions $g_{j}$ belong to $L^{2}(\Omega)$, then we have that $z_{\mu} \in L^{2}\left(0, T ; H_{0}^{1}(\Omega)\right) \cap L^{\infty}\left(0, T ; L^{2}(\Omega)\right)$; see [5] for details. We also observe that the above formulation of the control problem is very interesting because of the potential real-world applications.

Example 3. Our last example deals with a boundary parabolic control problem

$$
\begin{cases}\text { Minimize } & J(u):=\int_{0}^{T} \int_{\omega} L\left(x, t, y_{u}(x, t)\right) \mathrm{d} x \mathrm{~d} t \\ \text { such that } & \alpha(x, t) \leq u(x, t) \leq \beta(x, t) \quad \text { for a.a. }(x, t) \in \Sigma\end{cases}
$$

where $\alpha, \beta \in L^{\infty}(Q), \omega \subset \bar{\omega} \subset \Omega$ with $|\omega|>0$, and $y_{u}$ is the weak solution of the parabolic boundary value problem

$$
\left\{\begin{aligned}
\partial_{t} y+A y+a(\cdot, \cdot, y)=0 \quad & \text { in } Q=\Omega \times(0, T) \\
y(0)=y_{0} & \text { in } \Omega \\
\partial_{n_{A}} y & =u \quad \text { on } \Sigma=\Gamma \times(0, T) .
\end{aligned}\right.
$$

All the assumptions of the abstract framework are fulfilled if we take $X=\Sigma$ and $\eta$ is the usual measure on this lateral boundary of the cylinder $Q$. In order to deal with hypothesis (H9) we have assumed that the cost functional involves only the values of the states in the subdomain $\omega \times(0, T)$. The reason for this choice is that the solution $z_{\mu}$ of the linearized state equation corresponding to the measure $\mu \in \mathcal{M}(X)=\mathcal{M}(\Sigma)$ does not enjoy the $L^{2}(Q)$ regularity. Actually this solution belongs to the space $L^{p}\left(0, T ; W^{1, q}(\Omega)\right)$ for every $p, q \in[1,2)$ satisfying $\frac{1}{p}+\frac{n}{2 q}>\frac{1+n}{2}$; see [7]. However, $z_{\mu}$ is regular in interior subdomains $\omega \times(0, T)$ and (H9) holds in this case. We also observe that the fact of considering subdomains in the cost functional as done above is also quite frequent in control theory. 
4. Conclusions and outlook. In this paper, we have verified sufficient secondorder conditions for an optimal control problem with bang-bang controls under the structural assumption (2.16) on the adjoint state. The derivations have been made for an elliptic semilinear state equation, but the same technique can be used for other state equations provided that certain estimates on the control-to-state mapping are satisfied.

We did not address necessary conditions of second order. Indeed, it seems to be a delicate issue to derive such conditions based on the critical cone $\mathcal{T}_{\bar{u}}$, and this is subject to further research.

Finally, it would be interesting to see whether the quadratic growth in $L^{1}(\Omega)$ can be used to provide discretization error estimates similar to those of $[8,25]$ in the convex, linear-quadratic case.

\section{REFERENCES}

[1] W. Alt And M. Seydenschwanz, Regularization and discretization of linear-quadratic control problems, Control Cybernet., 40 (2011), pp. 903-920.

[2] F. Bonnans and A. Shapiro, Perturbation Analysis of Optimization Problems, SpringerVerlag, Berlin, 2000.

[3] H. Brezis, Functional Analysis, Sobolev Spaces and Partial Differential Equations, Springer, New York, 2011.

[4] E. CASAS, Boundary control of semilinear elliptic equations with pointwise state constraints, SIAM J. Control Optim., 31 (1993), pp. 993-1006, https://doi.org/10.1137/0331044.

[5] E. CASAS, Pontryagin's principle for state-constrained boundary control problems of semilinear parabolic equations, SIAM J. Control Optim., 35 (1997), pp. 1297-1327, https://doi.org/ $10.1137 /$ S0363012995283637.

[6] E. CASAS, Second order analysis for bang-bang control problems of PDEs, SIAM J. Control Optim., 50 (2012), pp. 2355-2372, https://doi.org/10.1137/120862892.

[7] E. Casas, J.-P. Raymond, And H. Zidani, Pontryagin's principle for local solutions of control problems with mixed control-state constraints, SIAM J. Control Optim., 39 (2000), pp. 1182-1203, https://doi.org/10.1137/S0363012998345627.

[8] K. Dechelnick And M. Hinze, A note on the approximation of elliptic control problems with bang-bang controls, Comput. Optim. Appl., 51 (2012), pp. 931-939, https://doi.org/10. 1007/s10589-010-9365-z.

[9] J. DunN, On second order sufficient optimality conditions for structured nonlinear programs in infinite-dimensional function spaces, in Mathematical Programming with Data Perturbations, A. Fiacco, ed., Marcel Dekker, New York, 1998, pp. 83-107.

[10] I. Ekeland and R. Témam, Convex Analysis and Variational Problems, Classics Appl. Math. 28, SIAM, Philadelphia, 1999, https://doi.org/10.1137/1.9781611971088.

[11] U. Felgenhauer, On stability of bang-bang type controls, SIAM J. Control Optim., 41 (2003), pp. 1843-1867, https://doi.org/10.1137/S0363012901399271.

[12] A. S. Kechris, Classical Descriptive Set Theory, Grad. Texts in Math. 156, Springer-Verlag, New York, 1995, https://doi.org/10.1007/978-1-4612-4190-4.

[13] H. Maurer and N. P. Osmolovski, Second order optimality conditions for bang-bang control problems, Control Cybernet., 32 (2003), pp. 555-584.

[14] H. Maurer and N. P. OsmolovskiI, Second order sufficient conditions for time-optimal bangbang control, SIAM J. Control Optim., 42 (2004), pp. 2239-2263, https://doi.org/10.1137/ S0363012902402578.

[15] H. Maurer AND J. Zowe, First and second order necessary and sufficient optimality conditions for infinite-dimensional programming problems, Math. Programming, 16 (1979), pp. 98110, https://doi.org/10.1007/BF01582096.

[16] C. Meyer, L. Panizzi, And A. Schiela, Uniqueness criteria for the adjoint equation in stateconstrained elliptic optimal control, Numer. Funct. Anal. Optim., 32 (2011), pp. 983-1007, https://doi.org/10.1080/01630563.2011.587074.

[17] A. A. Milyutin And N. P. Osmolovskit, Calculus of Variations and Optimal Control, Transl. Math. Monogr. 180, American Mathematical Society, Providence, RI, 1998.

[18] N. P. Osmolovskil, Quadratic conditions for nonsingular extremals in optimal control (a theoretical treatment), Russian J. Math. Phys., 2 (1994), pp. 487-516. 
[19] N. P. Osmolovskil And H. Maurer, Equivalence of second order optimality conditions for bang-bang control problems. I. Main results, Control Cybernet., 34 (2005), pp. 927-950.

[20] N. P. Osmolovski And H. Maurer, Equivalence of second order optimality conditions for bang-bang control problems. II. Proofs, variational derivatives and representations, Control Cybernet., 36 (2007), pp. 5-45.

[21] W. Rudin, Real and Complex Analysis, McGraw-Hill, London, 1970.

[22] G. Stampacchia, Le problème de Dirichlet pour les équations elliptiques du second ordre à coefficients discontinus, Ann. Inst. Fourier (Grenoble), 15 (1965), pp. 189-258.

[23] F. Tröltzsch, Optimal Control of Partial Differential Equations: Theory, Methods and Applications, Grad. Stud. Math. 112, American Mathematical Society, Providence, RI, 2010.

[24] V. M. Veliov, Error analysis of discrete approximations to bang-bang optimal control problems: The linear case, Control Cybernet., 34 (2005), pp. 967-982.

[25] D. Wachsmuth, Robust error estimates for regularization and discretization of bang-bang control problems, Comput. Optim. Appl., 62 (2015), pp. 271-289, https://doi.org/10.1007/ s10589-014-9645-0.

[26] D. Wachsmuth And G. Wachsmuth, Convergence and regularisation results for optimal control problems with sparsity functional, ESAIM Control Optim. Calc. Var., 17 (2011), pp. 858-886, https://doi.org/10.1051/cocv/2010027. 